\title{
Quantum Communication Networks
}

\author{
A Master of Science Thesis (M.Sc.) \\ Based on Experimental Work in The \\ QuANTUM InFormation AND QuANTUM Optics Group \\ KIKo \\ Department of Physics \\ Stockholm University
}

BY

NIMA RAFIEI

14 MAY 2008

SUPERVISOR

Dr. Mohamed Bourennane

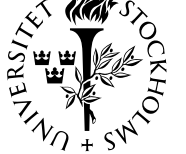

Stockholm

University 


\begin{abstract}
Quantum communication protocols invoke one of the most fundamental laws of quantum mechanics, namely the superposition principle which leads to the no-cloning theorem. During the last three decades, quantum cryptography have gone from prospective theories to practical implementations scalable for real communication. Scientist from all over the world have contributed to this major progress, starting from Stephen Wiesner, Charles H. Bennett and Gilles Brassard who all developed the theory of Quantum Key Distribution (QKD). QKD lets two users share a key through a quantum channel (free space or fiber link) under unconditionally secure circumstances. They can use this key to encode a message which they thereafter share through a public channel (internet, telephone,...). Research developments have gone from the ordinary 2-User Quantum Key Distribution over very small free space distances to distances over $200 \mathrm{~km}$ in optical fiber and Quantum Key Distribution Networks.
\end{abstract}

As great experimental achievements have been made regarding QKD protocols, a new quantum communication protocol have been developed, namely Quantum Secret Sharing. Quantum Secret Sharing is an extension of an old cryptography scheme called Secret Sharing. The aim of secret sharing is to split a secret amongst a set of users in such a way that the secret is only revealed if every user of this set is ready to collaborate and share their part of the secret with other users.

We have developed a 5-User QKD Network through birefringent single mode fiber in two configurations. One being a Tree configuration and the other being a Star configuration. In both cases, the number of users, the distances between them and the stability of our setup are all well competitive with the current worldwide research involving similar work.

We have also developed a Single Qubit Quantum Secret Sharing scheme with phase encoding through single mode fiber with 3, 4 and 5 parties. The latter is, to the best of our knowledge, the first time a 5-Party Single Qubit Quantum Secret Sharing experiment has been realized. 


\section{Kvantoptiska Kommunikations - Nätverk}

Kvantkryptisk kommunikation åberopar kvantmekanikens mest grundläggande lagar, nämligen superpositionsprincipen som leder till teoremet om icke-kloningsbarhet. De tre senaste årtionden, har kvantkryptografin utvecklats från lovande teorier till en realisering av kvantkommunikation utanför laborationsutrymmen. Forskare från hela världen har bidragit och tagit del av denna utveckling. Några som bör nämnas vid namn är Stephen Wiesner, Charles H. Bennett och Gilles Brassard vilka alla utvecklade den tidiga teorin av Quantum Key Distribution (QKD) (Kvantdelning av Nyckel). QKD delar en nyckel mellan två parter genom ovillkorligt säkra förhållanden. Denna nyckel kan användas av den ena parten för att koda ett meddelande som hon sedan skickar till den andre genom offentlig media (telefon, internet, etc...). Den andre parten kan då avkoda meddelandet med samma nyckel. I dagsläget har utvecklingen gått från en standard QKD med 2 parter med väldigt korta sträckor dem emellan till över $200 \mathrm{~km}$ genom optisk telekomfiber och QKD nätverk.

Teorin har också vidareutvecklats och lett till en ny kvantkryptisk kommunikationsprotokoll, nämligen Quantum Secret Sharing (QSS) (Kvantkryptisk Delning av Hemlighet). Quantum Ssecret Sharing är en utvidgning av en äldre klassisk kryptografisk protokoll, Secret Sharing (Delning av Hemlighet) vars mål är att dela en hemlighet bland en mängd parter på ett sådant sätt att den enbart kan rekonstrueras om alla parter väljer att samarbeta och dela deras del av hemligheten med resterande parter.

Vi har utvecklat en 5 User QKD Network genom dubbelbrytande optisk singel mod fiber i två olika konfigurationer, ena i en Träd - konfiguration och den andra i en Stjärn - konfiguration. I båda fallen är antalet parter, sträckorna mellan dem och stabiliteten av vår uppställning i forskningens framkant relativt andra forskningsframsteg runt om i världen.

Vi har också utvecklat en Single Qubit Quantum Secret Sharing uppställning med faskodning genom optisk fiber med 3, 4 och 5 parter. Detta framsteg är till vår kännedom den första 5 parters Kvantkryptisk Delning av Hemlighet uppställning som någonsin genomförts. 


\section{Acknowledgements}

I would like to show my gratitude to my supervisor dr. Mohamed Bourennane who is a great mentor and leader and who impersonates and radiates Nike's motto Impossible is nothing. I would like to thank my closest colleague during this project and also a mentor Jan Bogdanski, who introduced me to all components and electronics in the early days and who has managed to survive (not unharmed) our very loud and aggressive discussions. Also, I would like to thank the members of the KiKo - group, Hatim Azzouz, Magnus Rådmark, Stefan Holmgren and Piotr Badziag for their support and warm welcomes, and Elias Amselem who has been interrupted (more than once) in order to take part in answering my questions about these strange wave-like particles called photons (or particle-like waves...).

I would also like to show my gratitude to my family and friends who have been very supportive and considerate. I want to show special love and gratitude to my mother who have supported me and my quest beyond what I am able to put in words. But I will write a quote.

You say the words that can get me back in focus

Dear Mama, Tupac Shakur

Finally I would like to thank my beautiful wife who has been the one standing behind my success. I know you have been the one that had to deal with me during my cloudiest moments, but also during my happiest hours. I love you.

Sincerely yours

Nima 


\section{Contents}

Abstract

Kvantoptiska Kommunikations - Nätverk ii

Acknowledgements

Contents iv

1 Introduction 1

1.1 Key Distribution . . . . . . . . . . . . . . . 1

1.2 Quantum Key Distribution . . . . . . . . . . . . 2

1.3 Multi-User QKD Network . . . . . . . . . . . . . . 2

1.4 Quantum Secret Sharing . . . . . . . . . . . . . 3

1.5 Thesis contents .................... 3

I Secure Quantum Communication 4

2 Quantum Key Distribution $\quad 5$

2.1 Introduction . . . . . . . . . . . . . 5

2.2 Polarization Encoding . . . . . . . . . . . . . 6

2.3 Phase Encoding . . . . . . . . . . . . . . . . . 7

2.4 Protocol . . . . . . . . . . . . . . . . . . . 10

2.4 .1 One-Time Pad . . . . . . . . . . . . . . 10

2.5 Eavesdropping . . . . . . . . . . . . . . . . . 11

2.5.1 A brief analysis . . . . . . . . . . 11

3 Multi-User QKD Network $\quad 13$

3.1 Introduction . . . . . . . . . . . . . . . . 13

3.2 Protocol . . . . . . . . . . . . . . . . . . 14

3.2.1 Multi-User One-Time Pad . . . . . . . . . . . . 15 
4 Quantum Secret Sharing $\quad 16$

4.1 Introduction . . . . . . . . . . . . . . . . 16

4.2 Phase Encoding . . . . . . . . . . . . . . . . 16

4.3 Protocol . . . . . . . . . . . . . . . . . 18

$\begin{array}{ll}\text { II The Experiments } & 21\end{array}$

5 Quantum Information: Generating and Processing 22

5.1 Generation of Single Photons . . . . . . . . . . . . . 22

5.1.1 Faint Pulse and Average photon count, $\mu$. . . . . 22

5.2 Quantum Information Processing . . . . . . . . . . . . . 23

5.2 .1 Rate . . . . . . . . . . . . . . . 23

5.2 .2 Visibility . . . . . . . . . . . . . . . . . . 24

5.2 .3 Quantum Bit Error Rate . . . . . . . . . . . 25

6 Plug and Play Interferometric Setup With Phase Encoding 27

6.12 End-User Plug and Play QKD . . . . . . . . . . . 27

6.1 .1 Birefringence . . . . . . . . . . . . 27

6.1 .2 Setup . . . . . . . . . . . . . . 31

6.2 Multi-User Plug and Play QKD Network . . . . . . . . . . . 41

6.3 Plug and Play Quantum Secret Sharing . . . . . . . . . . 43

7 Experimental Components $\quad 45$

7.1 Pulse Generator . . . . . . . . . . . . . . . . . . 45

7.2 Laser Source . . . . . . . . . . . . . . . . . . . . 47

7.3 Digital Variable Attenuator . . . . . . . . . . . . . 47

7.4 Parties . . . . . . . . . . . . . . . . . 48

7.5 Fiber spools . . . . . . . . . . . . . . 48

7.6 Optical switches . . . . . . . . . . . . . . . . 49

7.7 Low-noise photo-diode . . . . . . . . . . . . . . . . 49

7.8 Single Photon Detectors . . . . . . . . . . . . 50

8 Experimental Results $\quad \mathbf{5 2}$

8.1 Multi-User QKD . . . . . . . . . . . . . . . . . . . . . . . 52

8.1 .1 Tree Configuration . . . . . . . . . . . . . 53

8.1.2 Star Configuration . . . . . . . . . . . . . 54

8.2 Quantum Secret Sharing . . . . . . . . . . . . . 55

8.2 .13 Parties..................... 55

8.2 .24 parties . . . . . . . . . . . . . . 58

8.2 .35 Parties . . . . . . . . . . . . . . 60

8.3 Discussion . . . . . . . . . . . . . . . 61 
III Conclusion

IV Appendix A

65

List Of Figures

List Of Tables

69

Bibliography

71 


\section{Chapter 1}

\section{Introduction}

\subsection{Key Distribution}

Transferring sensitive information between two points in a secure way which is not susceptible to eavesdropping is an attractive goal for all human networks. The means of the information transfer may consist of everything from a secret whisper, communication taking place in social gatherings, to the way intelligence agencies share information with their undercover agents in foreign countries. The most familiar way of security measures in our every day life is recognized through our ways of handling information over the internet, Personal Identification Number (PIN) codes, banking applications etc... .

Algorithm encoding is an ancient communication protocol which enables legitimate users to communicate in a secure way. The users only need to agree on an algorithm which they use to encode/decode a message. One of them uses the algorithm to encode a message and sends this encoded message through a public channel (internet, telephone...) to the others who then decode the message using the apriori agreed algorithm. Although this protocol seems quite easy and straightforward there exist one very important factor of weakness in terms of security. The time it takes for an eavesdropper to crack an algorithm is only limited by computational technology. Knowing this fact, one easily deduce that algorithms have a very short lifetime. This leads to an inconvenient procedure where legitimate users need to have personal meetings on regular bases in order to agree on a new algorithm, since communication through other means are highly susceptible to eavesdropping attacks.

A simple and unconditionally secure algorithm encryption uses the sum modulo 2 XOR operator of the two binary numbers 0 and 1 . Users distribute a Private Key through a personal meeting and keep this key secret. 
This apriori shared key, the Private Key, is used to lock (encode) a secret message through this XOR operator, see table 1.1. The encoded message is then announced through a public channel from one user to the other. The receiving user then uses the Private Key to unlock (decode) the encoded message and gain access to the secret message. However, this key distribution protocol with its secure XOR encryption algorithm still suffers from the previous mentioned setback that users need to personally meet each other in order to agree on a Private Key. We introduce the XOR operator in this section since it is the basic language of quantum communication protocols which themselves provide further improvements for an unconditionally secure method of sharing the Private Key.

\begin{tabular}{lccccc}
\hline \hline Private Key & & 0 & 1 & 0 & 1 \\
Secret message & + & 0 & 0 & 1 & 1 \\
\hline Encoded message & $=$ & 0 & 1 & 1 & 0 \\
\hline
\end{tabular}

Table 1.1: Sum modulo 2 XOR operation of the binary numbers 0 and 1.

\subsection{Quantum Key Distribution}

Quantum Key Distribution (QKD) is, as far as the protocol is concerned, identical to the classical key distribution protocol except for one part. To establish a Private Key, the two legitimate users use single quantum states as their binary bits. For instance, when the quantum state is a photon, horizontal polarization denotes binary bit 0 , and vertical polarization denote binary bit 1 . Now, when quantum states are assigned to binary bits, we then call them quantum bits, or qubits. The benefit of QKD is like many (if not all) quantum communication protocols. They all take advantage of a rule which states that a single unknown quantum state can not be perfectly cloned. This simply means that if an eavesdropper tries to intercept a qubit sent from one user to the other, clone it, and send the clone to the other user, her presence will be revealed since the clone will by statistics not be the one originally sent from its source each time.

\subsection{Multi-User QKD Network}

Multi-User QKD Networking follows the protocol and principles of ordinary QKD described in previous section, only here there are more users at hand. The users may be connected in different network configurations. In this Thesis we have implemented a one-to-many configuration in two different settings. One being a Tree configuration and one being a Star configuration. In a one-to-many configuration, there is one master connected to other so 
called end-users. The communication takes place between the master and one end-user at a time, hence the similarity to ordinary QKD.

\subsection{Quantum Secret Sharing}

Quantum Secret Sharing is a quantum version of a classical communication protocol called secret sharing. The aim of secret sharing is to split a secret amongst a set of parties ${ }^{1}$ where each part of the secret is useless as a standalone piece of information. The secret is only revealed when all parties decide to collaborate and reveal their part of the secret to other parties.

\subsection{Thesis contents}

We will present the theory of ordinary 2-End-User Quantum Key Distribution and extrapolate this to the Multi-User QKD Network, for which we will present our experimental results for the two 5-User QKD Network configurations. We will also present the theory of Quantum Secret Sharing as well as our experimental results for 3, 4 and 5 Party Single Qubit Quantum Secret Sharing with phase encoding implementation.

\footnotetext{
${ }^{1}$ Here we call the users parties instead, since all of the users take part of the protocol simultaneously.
} 


\section{Part I}

\section{Secure Quantum \\ Communication}




\section{Chapter 2}

\section{Quantum Key Distribution}

\section{$2.1 \quad$ Introduction}

Quantum key distribution is the name of a quantum communication protocol between two end users, called Alice and Bob. Alice is usually the sender and Bob is the receiver. A qubit (a quantum state carrying information) is prepared and sent from one user to the other. The transfer link might be free space link as well as optical fiber link. The idea is to have two such users communicating over a secure quantum channel which is immune to eavesdropping attacks. Security analysis of protocols usually assume the presence of an eavesdropper, Eve, with unlimited resources while limited by laws of physics, or rather laws of quantum mechanics. Quantum communication protocols take advantage of one of the most fundamental laws of quantum mechanics, namely the superposition principle which leads to the no-cloning theorem. The theorem basically states that a single unknown quantum state cannot be perfectly cloned (duplicated). This means that Eve cannot possibly (without prior knowledge about the qubit sent from Alice to Bob) gain knowledge about the qubit without introducing any disturbance and therefore revealing her presence.

The BB84 [1] from 1984 is one of the best known quantum key distribution protocols. The idea of this protocol is to let Alice and Bob share a key through a secure quantum channel. The key consist of string elements with bit values of 0 and 1, constituting a resourceful base for various representations, such as key-codes and text representation. In the following, we will present the BB84 protocol in practical details and analyze its further extensions. 


\section{$2.2 \quad$ Polarization Encoding}

For simplicity, let us first understand the polarization encoding implementation before moving on to the phase encoding one. It also follows the chronological order of these two implementations.

The idea of this implementation is to store information in the polarization state of a photon. Once a state is assigned to carry a specific piece of information e.g binary bits, 0's and 1's, we then call the (quantum state) photon carrying such a bit a quantum bit, or rather, a qubit. It has been shown [7] that two nonorthogonal states is sufficient enough to design a secure quantum cryptography encoding implementation, since they are then not unambiguously distinguishable upon measurement by an eavesdropper who will then inevitably induce errors which can be noticed by the legitimate users. However, in [8] the BB84 communication implementation is described by introducing a 4-state polarization encoding protocol whereas two of these states are represented through a rectilinear $(\mathrm{R})$ basis, $\{\mathrm{H}, \mathrm{V}\}$, with the encoding $0 \rightarrow|\mathrm{H}\rangle$ and $1 \rightarrow|\mathrm{V}\rangle$, and two through a diagonal (D) basis $\{+45,-45\}$, with the encoding $0 \rightarrow|+45\rangle$ and $1 \rightarrow|-45\rangle$ see fig. 2.1 and table 2.1. Of course, these two bases are related through linear combinations. In fact, that is the main intension, to keep the bases nonorthogonal, i.e $|\langle\mathrm{H} \mid+45\rangle|=|\langle\mathrm{H} \mid-45\rangle|=|\langle\mathrm{V} \mid+45\rangle|=|\langle\mathrm{V} \mid-45\rangle|=\frac{1}{\sqrt{2}}$.

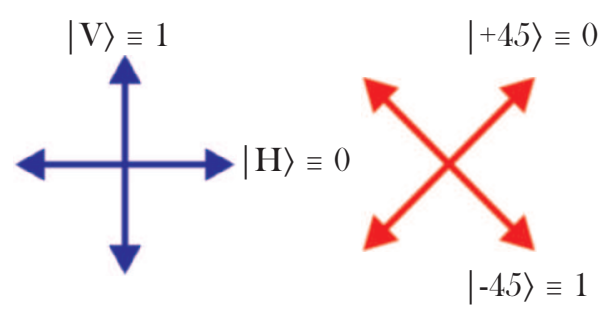

$\underline{\mathrm{R}}$

$\underline{\mathrm{D}}$

Figure 2.1: BB84 polarization encoding implementation, using four nonorthogonal onephoton states. 


\begin{tabular}{l|clllllll}
\hline \hline Alice sends qubit prepared in state & $\mathrm{R}$ & $\mathrm{R}$ & $\mathrm{D}$ & $\mathrm{D}$ & $\mathrm{R}$ & $\mathrm{R}$ & $\mathrm{D}$ & $\mathrm{D}$ \\
Bit value & 0 & 1 & 0 & 1 & 1 & 0 & 1 & 0 \\
Bob measures qubit values & 0 & 1 & 1 & 1 & 0 & 0 & 1 & 1 \\
Bob announce his choice of basis & $\mathrm{R}$ & $\mathrm{D}$ & $\mathrm{R}$ & $\mathrm{D}$ & $\mathrm{D}$ & $\mathrm{R}$ & $\mathrm{D}$ & $\mathrm{R}$ \\
Alice tell which basis are compatible & $\mathrm{Y}$ & $\mathrm{N}$ & $\mathrm{N}$ & $\mathrm{Y}$ & $\mathrm{N}$ & $\mathrm{Y}$ & $\mathrm{Y}$ & $\mathrm{N}$ \\
Bob filters his data & 0 & - & - & 1 & - & 0 & 1 & - \\
\hline Shared key & 0 & & & 1 & & 0 & 1
\end{tabular}

Table 2.1: BB84 polarization encoding protocol using four nonorthogonal one-photon states. Announcement is made through a public channel.

It is of utmost importance to understand the BB84 polarization encoding protocol, i.e. the procedure described in table 2.1, since the phase encoding protocols [7] is derived from to this protocol.

\subsection{Phase Encoding}

The phase encoding protocol (B92) was proposed in 1992 by C.H. Bennett [7]. The phase encoding implementation was used in the experimental part of this Thesis. Here we discuss the protocol in detail and it will be quite clear why this is the preferred protocol rather than the polarization encoding protocol.

\section{It is indeed a very natural choice for optics specialists.[5]}

The idea of this protocol is to store information in the relative phase difference between two pulses. State preparation is rather simple and since the information itself is then polarization-independent, this protocol can easily be realized with the usage of single mode fibers, over longer fiber links. Since the interferometer is to measure the relative phase difference, it is suggested to use an optical fiber version of a Mach-Zehnder interferometer. First we can view and recall a standard free space Michelson interferometer in fig. 2.2 , where the intensity $I$ is a function of the path length difference. This setup demands that the coherence length of the input light is at least longer than the path length difference. Even with a large, but finite coherence time, one will occasionally notice disturbances on the expected Gaussian plot. A Mach-Zehnder interferometer, on the other hand, have nearly no demand on the coherence time but it instead rely on perfect calibration and extremely stable setup, i.e $\left\{A_{1}, A_{2}\right\}=\left\{B_{2}, B_{1}\right\}$ see fig. 2.3. This interferometer instead, couples the two mirrors in fig. 2.2 through a beam splitter (BS). 


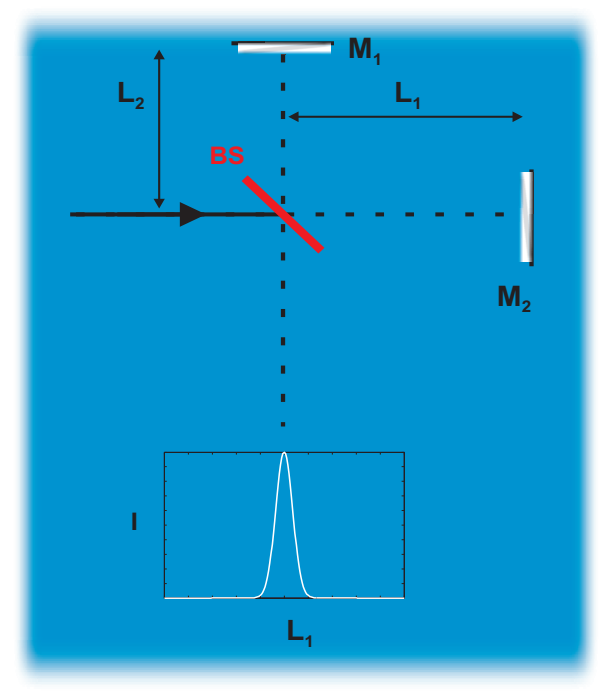

Figure 2.2: A setup of a free space Michelson interferometer. The sub-figure is showing the intensity as a function of the distance from the beam splitter to the mirror $M_{2}$, i.e $L_{1}$, (when $L_{2}$ is kept constant). Of course, for continuous light, there are infinite number of peaks as $L_{1}$ increases.

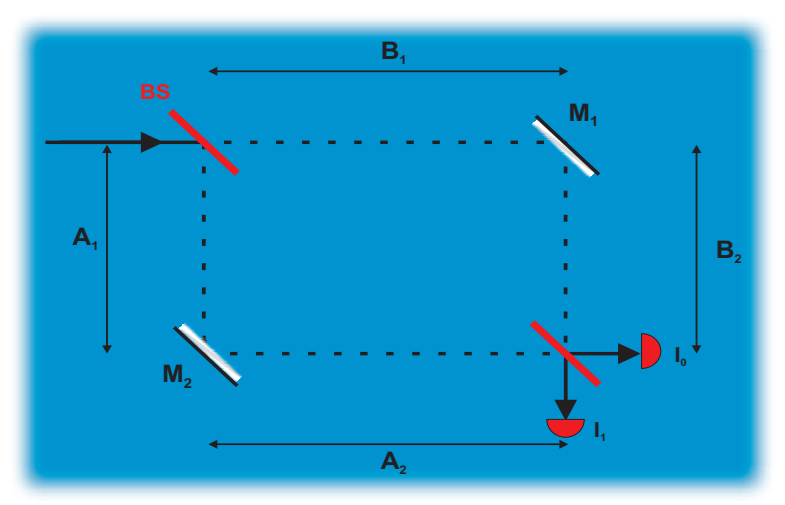

Figure 2.3: A freespace Mach-Zehnder interferometer. BS, beam splitter; I, intensity detector.

We can analyze an optical fiber version of a Mach-Zehnder interferometer in fig. 2.4 (the used interferometer will be discussed in detail in ch. 6), which is used in a standard B92 4-state phase encoding protocol. For the time being, let us assume that the polarization is maintained throughout the fiber link by using polarization maintaining fiber or PBC's (Polarization Beam Controllers), (we will discuss this polarization problem in ch. 6 Plug and Play). Consider fig. 2.4. A pulse is sent from a laser diode (LD). The pulse passes a beam splitter (BS). The pulse traveling along the lower arm is phase modulated by Alice with a relative phase difference of $\phi_{A}$. 
The pulse traveling along the upper arm is phase modulated by Bob with a phase shift of $\phi_{B}$, before the two pulses interfere when passing through the $2 \times 2$ BS just to hit one of the two detecting APD's (avalanche photo diode) located at Bob's station, (notice a $\pi / 2$ phase shift experience upon reflection through a $2 \times 2 \mathrm{BS}$, relative transmission. This is carefully analyzed in section 6.1.2 Setup - Alice, the interferometer). Now, the APD's are assigned the bit values 0 and 1 . Let $I_{s}$ denote the $\mathrm{LD}$ intensity (assume negligible attenuation in fiber), then the intensities measured at the APD's are given by eq. 2.1 and eq. $2.2[5]$,

$$
\begin{aligned}
& I_{1}=I_{s} \cos ^{2}\left(\frac{\phi_{A}-\phi_{B}+k \Delta L}{2}\right) \\
& I_{0}=I_{s} \sin ^{2}\left(\frac{\phi_{A}-\phi_{B}+k \Delta L}{2}\right)
\end{aligned}
$$

where $\mathrm{k}$ is the wave number $\left(2 \pi \lambda^{-1}\right)$, and $\Delta L$ is the path length difference.

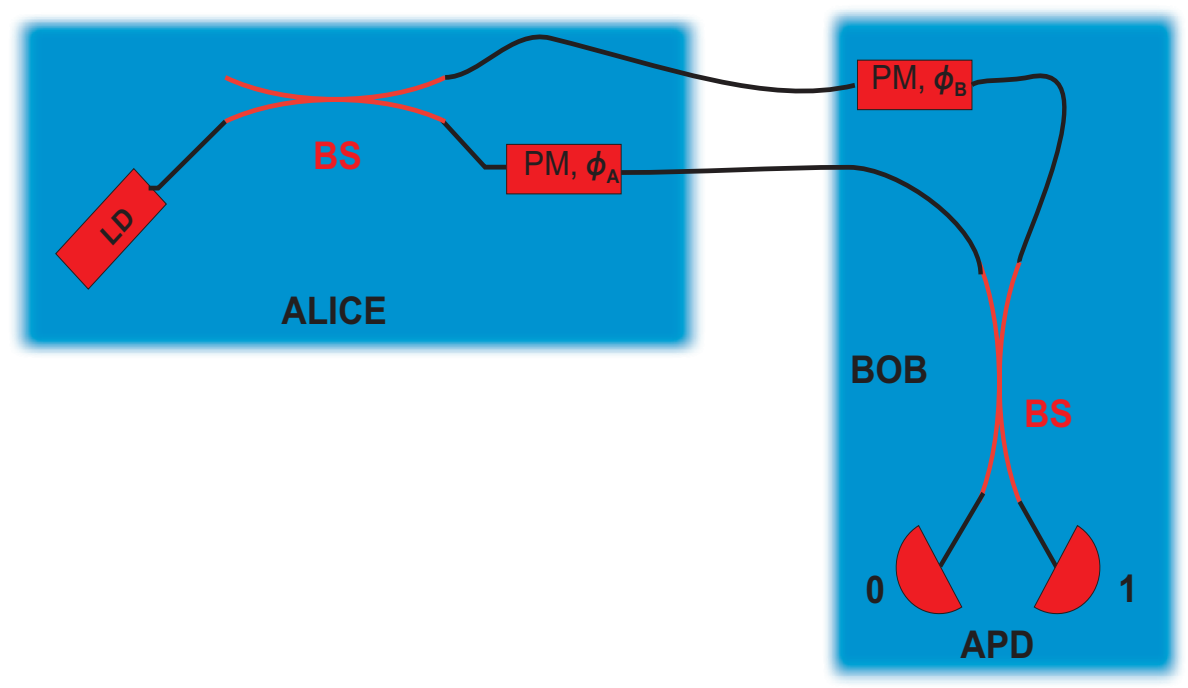

Figure 2.4: QKD setup for the B92 4-state phase encoding protocol

Let us now assume that $\Delta L=0$. Alice will choose to phase modulate through one of the 4 phases $\{0, \pi\}$ and $\{\pi / 2,3 \pi / 2\}$. Bob, on the other hand, will only choose between $\{0, \pi / 2\}$, setting basis for measurements.

Once the quantum communication is over, Alice and Bob need to correlate this quantum information over a public channel. They both know that Bob's two bases in the set $\{0, \pi / 2\}$ are compatible with Alice's phase shifted qubit whenever the base $\{0\}$ is set for a qubit in the set $\{0, \pi\}$ and the base $\{\pi / 2\}$ for a qubit in $\{\pi / 2,3 \pi / 2\}$. Bob announces his set of base through a 
public channel and Alice then announces which of the bases that were compatible with her phase shifts. Now, Bob can just read of the values detected on the APD's and since Alice knows what qubit she prepared for a certain compatible base, she knows the deterministic result of Bob's measurement on the qubit in that state. See table 2.2, for some examples.

For quantum states, the intensities in eq. 2.1 and eq. 2.2 correspond instead to the probability, $P\left(I_{1}\right)$ and $P\left(I_{0}\right)$, that detector " 1 " and "0" count a photon, respectively. $I_{s}=1$ in this case.

\begin{tabular}{r|r|r|r|r|r|r}
\hline \hline \multicolumn{2}{c|}{ Alice } & \multicolumn{5}{c}{ Bob } \\
\hline Bit sent & $\phi_{A}$ & $\phi_{B}$ & $\phi_{A}-\phi_{B}$ & Bit detected & $P\left(I_{1}\right)$ & $P\left(I_{0}\right)$ \\
\hline 0 & 0 & 0 & 0 & 0 & 1 & 0 \\
\hline 0 & 0 & $\pi / 2$ & $3 \pi / 2$ & $?$ & $1 / 2$ & $1 / 2$ \\
\hline 1 & $\pi$ & 0 & $\pi$ & 1 & 0 & 1 \\
\hline 1 & $\pi$ & $\pi / 2$ & $\pi / 2$ & $?$ & $1 / 2$ & $1 / 2$ \\
\hline 0 & $\pi / 2$ & 0 & $\pi / 2$ & $?$ & $1 / 2$ & $1 / 2$ \\
\hline 0 & $\pi / 2$ & $\pi / 2$ & 0 & 0 & 1 & 0 \\
\hline 1 & $3 \pi / 2$ & 0 & $3 \pi / 2$ & $?$ & $1 / 2$ & $1 / 2$ \\
\hline 1 & $3 \pi / 2$ & $\pi / 2$ & $\pi$ & 1 & 0 & 1 \\
\hline
\end{tabular}

Table 2.2: The B92 4-states phase encoding protocol

\subsection{Protocol}

\subsubsection{One-Time Pad}

The One-Time pad [5] protocol provide a secure way for two end users to share a secret key. This key can have different purposes. The key itself could be e.g the PIN code for a joint bank account or to open a security lock. It could also be used to decode an encoded message sent from one user to another through a public channel. Although this key is shared in a secure way, it should only be produced and used once. Let us say that Alice and Bob establish a shared secret key which we call a reference key, $k_{r e f}$, through the phase encoding implementation mentioned in previous section. We call it a reference key since it can serve different purposes. Alice can then use this reference key to encode a secret message, $M_{\text {sec }}$, through the operation $M_{\text {sec }} \oplus k_{\text {ref }}=M_{\text {enc }}$ (let $\oplus$ denote a XOR operator such as sum modulo 2, see table 2.3). Alice then announces this encoded message, $M_{e n c}$, through a public channel to Bob. Bob can then decode the message by using the shared reference key, through the operation $M_{e n c} \oplus k_{r e f}=M_{s e c} \oplus k_{r e f} \oplus k_{r e f} \rightarrow M_{s e c}$, (since $k_{r e f} \oplus k_{r e f}=\{0\}$ ). Let us now analyze the case where this unique reference key is used twice. Consider two secret messages, $M_{s e c}^{1}$ and $M_{s e c}^{2}$, 
and two publicly announced encoded messages, , $M_{e n c}^{1}$ and $M_{e n c}^{2}$ belonging to the respective secret messages through the upper index. Eve can then perform the operation $M_{e n c}^{1} \oplus M_{e n c}^{2}=\left(M_{s e c}^{1} \oplus k_{r e f}\right) \oplus\left(M_{s e c}^{2} \oplus k_{r e f}\right)=$ $M_{\text {sec }}^{1} \oplus M_{\text {sec }}^{2} \oplus k_{\text {ref }} \oplus k_{\text {ref }} \Rightarrow M_{\text {sec }}^{1} \oplus M_{\text {sec }}^{2}$ (since, $\oplus$ is also commutative) and gain information about the sum of the two secret messages. And since we want Eve to have as little knowledge as possible about the information, we certainly do not want her to have information in this manner either, even if the information is encrypted in a sense.

\begin{tabular}{llllll}
\hline \hline Binary Bit, $k_{r e f}$ & & 0 & 1 & 0 & 1 \\
Binary Bit, $M_{s e c}$ & $\oplus$ & 0 & 0 & 1 & 1 \\
\hline Binary Bit, $M_{e n c}$ & $=$ & 0 & 1 & 1 & 0
\end{tabular}

Table 2.3: Sum modulo 2 operation

\subsection{Eavesdropping}

Analysis of quantum cryptographic protocols always assume the presence of an eavesdropper, who by convention is called Eve. Eve is assumed to have financially and technologically unlimited resources, creating worst nightmare conditions for other legitimate users. Also, her resources are not assumed to be limited by today's technology, unlike the legitimate users, Alice and Bob. In order for the analysis to be realistic and somewhat fair, Eve is assumed to be restricted by laws of quantum mechanics. The no-cloning theorem includes statistical and uncertainty principals of quantum states, which are to be regarded as the primary factors resulting in revealing Eve's presence.

\subsubsection{A brief analysis}

While a 2-states protocol has been proved [7] to be sufficient in order to create secure quantum cryptographic protocol, let us consider a 4-state protocol, with horizontal polarization $|H\rangle$, vertical polarization $|V\rangle$, linear polarization at $45^{\circ}|+45\rangle$ and linear polarization at $-45^{\circ}|-45\rangle$. Let the states $\{|H\rangle,|+45\rangle\}$ represent binary bit 0 and the states $\{|V\rangle,|-45\rangle\}$ represent binary bit 1 . The requirement, in order for the protocol to be secure, is that the states from each basis are non-orthogonal to the states from the other basis.

Let Alice be the sender of the quantum state and Bob be the receiver and regard only the cases when they choose correlated basis, since they will disregard other cases. In a so called intercept-resend attack [5], Eve will randomly choose, to perform a measurement in one of the basis, $\mathrm{R}$ 
$\{x, y\}=\{H, V\}$ or $\mathrm{D}\{x, y\}=\{+45,-45\}$ and copy the state she measured and send it to Bob. Now, in half of the cases she will have chosen the right basis, i.e compatible with the prepared state by Alice, and send the copy to Bob. In the other half, Eve will have chosen the wrong basis, and send a state incompatible with Bob's measuring base. However, there still exists a probability of $1 / 2$ that Bob will measure the qubit Alice has sent. So, when Alice and Bob decide to spend a portion of the key to check the presence of an eavesdropper, they will conclude that they have en error of $25 \%$ of the cases when they had been using correlated basis. Hence, Eve has revealed her presence and the two legitimate users, Alice and Bob will disregard the whole established key. To summarize, using 4 non-orthogonal states, Eve will gain $50 \%$ of the information and introduce $25 \%$ error with the interscept-resend attack. There are of course other methods through which Eve can choose to attack, such as photon-number-splitting (PNS) attacks which means that Eve uses a beam splitter to take one photon from one of the multiphoton pulses ${ }^{1}$ for herself and transmit the other to Bob. She then waits until Bob have announced his basis, and then performes a measurement in that very basis. The efficiency of this attack can be controlled by Alice and Bob by determining their average photon number $\mu^{2}$, in cost of information rate $(\mathrm{B} / \mathrm{s})$ exchanged between themselves. But note that Eve can always change the interconnecting fiber between Alice and Bob with an ideal lossless channel. If the probability of having a multiphoton pulse is larger than the probability that Bob is going to detect a non-empty pulse, then Eve can get full information without introducing any perturbation [5]. Another method of attack is to have Eve using a universal quantum cloning machine (UQCM) [16]. The UQCM enables Eve to clone a photon with unequal fidelity. She transmits the photon with higher fidelity to Bob and keep the photon with lower fidelity for herself. For identical clones the fidelity is given by $F=\frac{5}{6}$ and hence the disturbance is given by $D=1-F=\frac{1}{6}$

It has been shown in [15] that shared keys with errors above $11 \%$ are to be disregarded, since they no longer can be trusted to be secure. That is why our experimental data presented are all limited by this number.

\footnotetext{
${ }^{1}$ When attenuating classical light to single photon level, there will be a non-zero probability of having a pulse containing more than one single photon. We will analyse this effect in ch. 5 .

${ }^{2} \mu$ is the average number of photons in each pulse.
} 


\section{Chapter 3}

\section{Multi-User QKD Network}

\subsection{Introduction}

Here we extend the 2-end-user QKD to a N-user QKD implementation. The derivation is quite straightforward since the security analysis of the standard 2-user QKD apply to this implementation. The reason for this applicability is due to the fact that only one 2-end-user QKD communication is allowed at a time i.e a qubit travels along a quantum channel connecting two users and is hence encoded by these two end-users only, where one is Alice who is controlling the interferometer, and all other users (Bob, Charlie, David and Elisabeth) are connected to her through independent fiber links, as described in previous part. The experimental part of the thesis considers two network setups. One is a tree-like configuration, where users are aligned as in fig. 3.1. The other is a star-like configuration as shown in fig. 3.2. In fig. 3.1 it is obvious that the fiber links connecting Alice $\rightarrow\{$ David,Elisabeth $\}$ are not completely independent of the fiber link Alice $\rightarrow$ Charlie. However, this does not violate any previous assumptions. Optical switches are guiding the qubits to each user. 


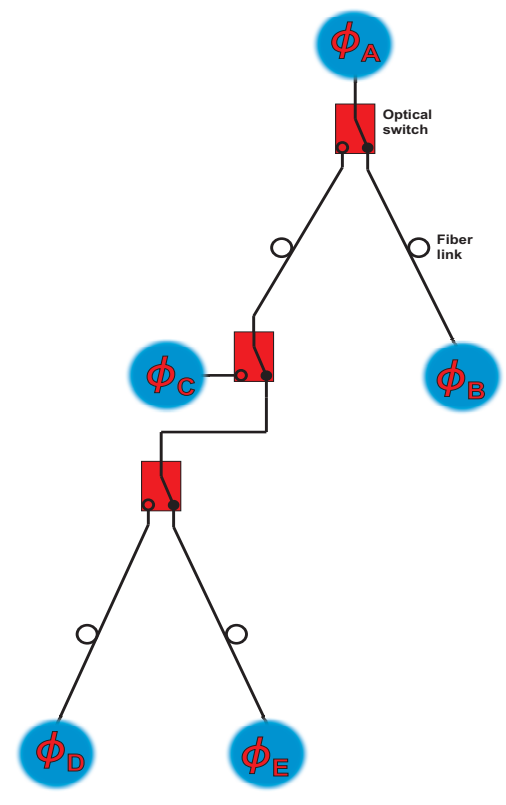

Figure 3.1: Tree configuration

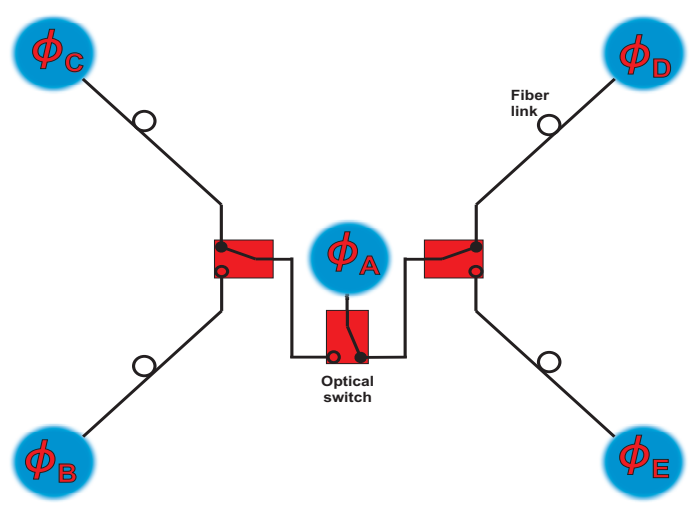

Figure 3.2: Star configuration

\subsection{Protocol}

It could not be more straightforward to extend the 2-user QKD One-Time Pad protocol to a N-user protocol since we have not altered the setup in any deviant way but adding users. In the following, we will present a $\mathrm{N}$-user protocol. 


\subsubsection{Multi-User One-Time Pad}

Assume that we have $\mathrm{N}$ users. Say Alice possesses a secret, $k_{\text {sec }}$, she wants to share with $\mathrm{N}-\mathrm{m}$ users $(m \geq 1)$. The first step is to establish a unique random reference key, $k_{r e f, j}$, where $j \in\{N-m\}$, with each user $j$, in accordance with the BB84 QKD One-Time Pad protocol. Alice then announces, through a public channel, a decryption key $k_{d e c, j}$, adapted uniquely for each $k_{r e f, j}$ in such a way that $k_{r e f, j} \oplus k_{d e c, j}=k_{s e c}$.

Both of the One-Time Pad protocols, as we have presented them, serves Alice when she wants to share her secret with selected users. Also, users are independent of each other i.e they do not have to collaborate during the protocol in order to gain knowledge about the secret. However, it should be emphasized that nothing prohibits users to use the reference key to encode a message and send this to Alice. 


\section{Chapter 4}

\section{Quantum Secret Sharing}

\subsection{Introduction}

Suppose...that a launch sequence of a nuclear missile is protected by a secret code. Yet, it should be ensured that a single lunatic alone is not able to activate it, but at least two lunatics are required. [17]

The aim of classical secret sharing is to split information in such a way that these pieces of split information is not enough to reconstruct any part of the secret itself. It can only be reconstructed if all of the pieces are put together. However, as in the case for a standard non-quantum key distribution, the security of this information splitting which takes place with help of classical communication, is susceptible to eavesdropping and its encoding is only limited by computational power. Quantum Secret Sharing suppress eavesdropping efficiency in the same manner as discussed in previous sections.

\subsection{Phase Encoding}

Theories for Quantum Secret Sharing are developed using entangled photon states e.g Greenberger - Horne - Zeilinger $|G H Z\rangle$ states [18] [19] sent to each party. Although the theory of using e.g a $|\psi\rangle=\frac{1}{\sqrt{2}}(\underbrace{|000 \ldots 0\rangle}_{N}+|111 \ldots 1\rangle)$ entangled state for $\mathrm{N}$ parties seems quite elegant with the correlation function $E\left(\phi_{1}, \phi_{2}, \ldots, \phi_{N}\right)=\cos \left(\phi_{1}+\phi_{2}+\ldots+\phi_{N}\right)$ where $\phi_{i}$ is the phase setting of each party, an experimental realization of these state is yet to become as elegant. An experimental four party quantum secret sharing using entangled photon states has been realized [20], on optical tables. However, rates and visibilities ${ }^{1}$ are not really comparable with our setup. A new protocol, based on single qubit communication has been introduced in [17], see fig.

\footnotetext{
${ }^{1}$ Rate and visibility are discussed in ch. 5 .
} 
4.1. It has been shown that this protocol gives the same correlation function as the protocol using $|G H Z\rangle$ states. Each party $\{N-1\}$ choose to encode the qubit by phase modulating the qubit through one of the four phases $\phi=\{0, \pi\}$ belonging to the base $\{0\}$, and $\phi=\{\pi / 2,3 \pi / 2\}$ belonging to the base $\{\pi / 2\}$, whereas the last party sets the basis and measures the qubit. This procedure is quite analogous to previous discussions, where the final state was denoted $\phi_{B}-\phi_{A}$. But here instead, it becomes $\left(\sum_{i=1}^{N-1} \phi_{i}\right)-\phi_{N}$. Some examples can be seen in table 4.1.

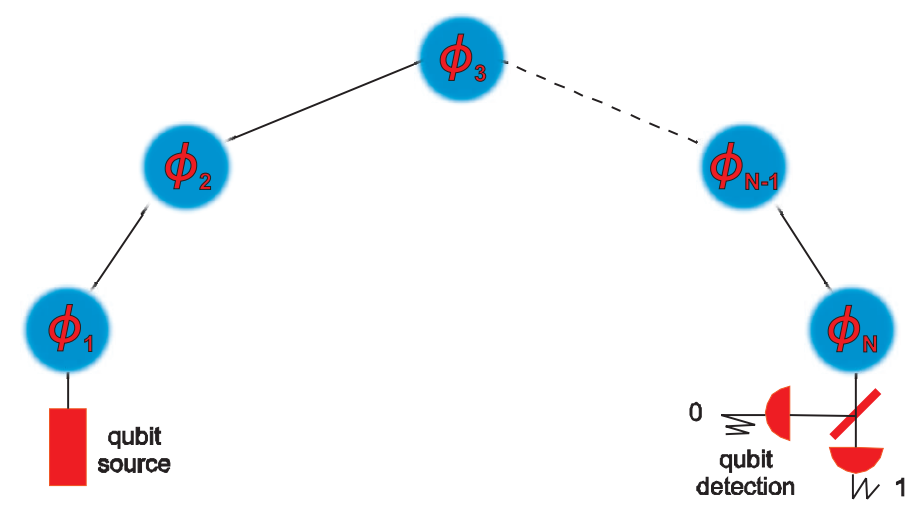

Figure 4.1: Single qubit secret sharing using phase encoding.

For simplicity, let us analyze a three party secret sharing phase encoding protocol. We can, as before, assign qubit values $\{0,1\} \rightarrow\{[0, \pi / 2],[\pi, 3 \pi / 2]\}$.

\begin{tabular}{ccccc}
\hline \hline 1 & 2 & 3 & $\left(\sum_{i=1}^{2} \phi_{i}\right)-\phi_{3}$ & Bit measured \\
\hline 0 & 0 & 0 & 0 & 0 \\
0 & 0 & $\pi / 2$ & $3 \pi / 2$ & $?$ \\
$\pi$ & 0 & 0 & $\pi$ & 1 \\
$\pi$ & 0 & $\pi / 2$ & $\pi / 2$ & $?$ \\
$\pi$ & $\pi / 2$ & 0 & $3 \pi / 2$ & $?$ \\
$\pi / 2$ & $\pi / 2$ & 0 & $\pi$ & 1 \\
$3 \pi / 2$ & $\pi$ & $\pi / 2$ & 0 & 0 \\
\hline
\end{tabular}

Table 4.1: A three party (1,2 and 3) table over some examples of qubit encoding and measurement results.

After a sequence, parties reveal their set of basis but keep the encoding phase, secret for themselves. For instance, consider the last row in table 4.1. Parties will announce (starting with $\phi_{3}$ ) the basis $\{\pi / 2, \pi, \pi / 2\}$, since phase encodings $\{0, \pi\}$ belong to base $\{0\}$ and $\{\pi / 2,3 \pi / 2\}$ belong to base $\{\pi / 2\}$. In this way, they will know that this particular sequence led to a valid and deterministic measurement on the last party's detectors. However, 
the subset ( party 1 and 2 ) does yet not know the actual result on party 3 's detectors. The results are only revealed to the subset if they choose to collaborate and reveal their choice of modulation (i.e the actual phase encoding).

\subsection{Protocol}

We have already discussed the core of the Secret Sharing phase encoding protocol. However, in previous discussions it is not clear how the parties should collaborate. Obviously, they could make their final announcement, in which the secret is revealed, through a personal meeting since public channels are not secure. Here we present a protocol which makes it possible for the parties to collaborate in a secure way with help from the master Alice.

\section{Multiparty Secret Sharing One-Time Pad}

Say Alice wants to share a secret, $k_{\text {sec }}$, among a subset $\{N-m\}$ of parties, in such a way that all parties among this subset must collaborate in order to reveal the secret (there is of course no real demand that this secret must have been generated through the Secret Sharing phase encoding implementation). As discussed in the QKD One-Time Pad, Alice establish a random reference key, $k_{r e f, j}$, through the quantum channel with each party $j$ of the subset, i.e $j \in\{N-m\}$, but this time Alice does not reveal a unique decryption key to these parties which would lead them to the secret, since a collaboration is required. Instead she publicly announce a decryption, $k_{d e c, j}$ to all parties, where these decryption keys are unique in the sense that a new subset of parties $n \in\{N-m \mid n \neq j\}$ can perform the operation $k_{r e f, n} \oplus k_{d e c, n}=k_{d e c, n}^{j}$. These decryption keys (denote missing links) are the ones party $j$ need in order to reveal the secret for himself. So when all of them are willing to collaborate, they announce this decryption key to party $j$ who then perform the operation $\sum^{n} k_{d e c, n}^{j}=k_{d e c, j}^{\prime}$ and finally $k_{d e c, j}^{\prime} \oplus k_{r e f, j}=k_{s e c}$.

Since a reference key cannot, in general, be uniquely adapted for parties to reveal the secret and to decrypt missing links for other parties. Each party $j$ need to have $\mathrm{N}-\mathrm{m}$ different reference keys. N-m-1 of them are used to decode other parties' missing links and one of them is used to reveal the secret for himself. However, even if this adaptation would be possible, it can easily be shown that the secret would then be revealed to Eve as mentioned in the original One-Time Pad. 


\section{Eavesdropping}

Since this protocol is based on the original One-Time Pad, along with it comes its drawback. Each party $j$ need to have N-m different reference keys. N-m-1 of them are used to decode other parties' missing links and one of them is used to reveal the secret. If they were to use the same reference key for all operations, Eve would be able to reveal the secret, given that she know the operator. Eve listens to all publicly announced keys i.e she knows $k_{d e c, j}^{\prime}$, since all $k_{d e c, n}^{j}$ are announced publicly. So, when party $j$ uses his reference key to decode his part of the decryption key for party $j-1$ (and also to reveal the secret for himself), he uses the publicly announced $k_{d e c, j}$, which is a unique decryption key for party $j$ to perform the operation $k_{r e f, j} \oplus k_{d e c, j}=k_{d e c, j}^{j-1}$, and publicly announce it to party $j-1$. Eve can then perform the operation, $k_{d e c, j}^{j-1} \ominus k_{d e c, j}=k_{r e f, j} \oplus k_{d e c, j} \ominus k_{d e c, j}=k_{r e f, j}$, and finally, $k_{r e f, j} \oplus k_{d e c, j}=k_{s e c}$.

\section{Example for three parties}

Say Alice posses a $k_{s e c}$ she wants to distribute to Bob and Charlie. She establishes two random reference keys with each party, $k_{r e f, B o b 1}, k_{r e f, B o b 2}$ and $k_{r e f, C h a r l i e 1}, k_{r e f, C h a r l i e}$. She publicly announces a $k_{d e c, C h a r l i e 1}$ to Charlie and a $k_{d e c, B o b 1}$ to Bob. Charlie performs the operation $k_{r e f, C h a r l i e 1} \oplus$ $k_{d e c, \text { Charlie } 1}=k_{\text {dec,Charlie } 1}^{\text {Bob }}$ and Bob, $k_{r e f, B o b 1} \oplus k_{d e c, B o b 1}=k_{d e c, B o b 1}^{\text {Charlie }}$. When they are willing to collaborate, Charlie publicly announce $k_{\text {dec,Charlie } 1}^{\text {Bob }}$ to Bob, and Bob publicly announce $k_{d e c, B o b 1}^{\text {Charlie }}$ to Charlie. They then perform the operation, (Charlie) $k_{\text {dec,Bob1 }}^{\text {Charlie }} \oplus k_{\text {ref,Charlie } 2}=k_{\text {sec }}$ and (Bob) $k_{\text {dec,Charlie } 1}^{B o b} \oplus k_{r e f, B o b 2}=k_{s e c}$. A numerical example is shown in table 4.2 and the calculations are shown below.

\begin{tabular}{llllll}
\hline \hline Secret & $k_{\text {sec }}$ & 1 & 0 & 1 & 1 \\
\hline Random ref. key & $k_{r e f, B o b 1}$ & 1 & 1 & 0 & 1 \\
\hline Random ref. key & $k_{r e f, B o b 2}$ & 1 & 0 & 1 & 0 \\
\hline Random ref. key & $k_{r e f, C h a r l i e 1}$ & 0 & 1 & 0 & 1 \\
\hline Random ref. key & $k_{r e f, C h a r l i e 2}$ & 1 & 0 & 0 & 1 \\
\hline Alice announce (to Bob) & $k_{\text {dec,Bob1 }}$ & 1 & 1 & 1 & 1 \\
\hline Alice announce (to Charlie) & $k_{\text {dec,Charlie } 1}$ & 0 & 1 & 0 & 0 \\
\hline
\end{tabular}

Table 4.2: Table over an example of the Multi-party Secret Sharing One-Time Pad

$$
\left\{\begin{array}{l}
k_{d e c, B o b 1} \oplus k_{r e f, B o b 1}=0010=k_{\text {dec }, \text { Bob } 1}^{\text {Charlie }} \\
k_{\text {dec }, \text { Charlie1 } 1} \oplus k_{\text {ref }, \text { Charlie1 } 1}=0001=k_{\text {dec }, \text { Charlie1 }}^{\text {Bob }}
\end{array} \Rightarrow\right.
$$




$$
\left\{\begin{array}{l}
k_{\text {dec }, \text { Bob } 1}^{\text {Charlie }} \oplus k_{\text {ref }, \text { Charlie } 2}=1011=k_{\text {sec }} \\
k_{\text {dec }, \text { Charlie } 1}^{\text {Bob }} \oplus k_{\text {ref }, \text { Bob } 2}=1011=k_{\text {sec }}
\end{array}\right.
$$




\section{Part II}

\section{The Experiments}




\section{Chapter 5}

\section{Quantum Information: Generating and Processing}

\subsection{Generation of Single Photons}

Since single photon sources are still in the stage of research and development and unavailable on any market, we have to attenuate classical coherent light (with Poisson distributed photon number) in such a way so that we at least have a Poisson distributed single photon source with a non zero probability of having more than one single photon in a pulse, (see sec. 5.1.1). This, is of course a disadvantage since quantum communication protocols rely on single qubits. In a multi-photon pulse, Eve can keep a photon for herself and let the other one be transmitted, a so called PNS attack. The attenuation $\mathrm{A}$, given in decibel with unit $\mathrm{dBm}$, is related to the power $\mathrm{P}$, given in unit of watt, through $A=10 \log _{10}\left(\frac{P}{1 m W}\right)$. See also app. A.

\subsubsection{Faint Pulse and Average photon count, $\mu$}

For the no-cloning theorem to have any meaning, one needs to keep the probability of having more than one photon per pulse, as low as possible. Obviously, the lower probability for a multi-photon pulse, the more information rate $(\mathrm{B} / \mathrm{s})$ one loses due to attenuation in the quantum channel (fiber). The average number of photons per pulse is denoted $\mu$, and its commonly acceptable values for quantum key distribution protocols are 0.1, 0.2 and at the extreme, 0.3. Since the number of photons in each pulse is Poisson distributed [5], see eq. 5.1 below,

$$
P(n)=e^{-\mu} \frac{\mu^{n}}{n !}
$$

the probability of having more than one photon in a weak non - empty pulse (faint pulse) i.e a multiphoton pulse is given by, 


$$
P(n>1)=\frac{1-P(n=0)-P(n=1)}{1-P(n=0)}
$$

where the divisor $1-\mathrm{P}(\mathrm{n}=0)$ denotes that only the non-empty pulses are included.

$$
\begin{gathered}
\left\{\begin{array}{l}
P(n=0)=e^{-\mu} \\
P(n=1)=\mu e^{-\mu} \\
e^{-\mu}=1-\mu+\frac{\mu^{2}}{2}+O\left(\mu^{3}\right)
\end{array} \quad \Rightarrow P(n>1)=\frac{1-\left(1-\mu+\frac{\mu^{2}}{2}+O\left(\mu^{3}\right)\right)(1+\mu)}{\mu-\frac{\mu^{2}}{2}+O\left(\mu^{3}\right)}=\right. \\
\quad=\frac{1-1-\mu+\mu+\mu^{2}-\frac{\mu^{2}}{2}+O\left(\mu^{3}\right)}{\mu-\frac{\mu^{2}}{2}+O\left(\mu^{3}\right)}=\frac{\frac{\mu^{2}}{2}+O\left(\mu^{3}\right)}{\mu-\frac{\mu^{2}}{2}+O\left(\mu^{3}\right)} \rightarrow \frac{\mu}{2-\mu} \approx \frac{\mu}{2}
\end{gathered}
$$

So the probability of having more than one photon in a weak non - empty pulse (faint pulse) is given by,

$$
P(n>1)=\frac{\mu}{2}
$$

\subsection{Quantum Information Processing}

After a quantum communication channel has been established, which in our case is optical single mode fiber, one have to start analyzing the information through the quantum channel. This is done by investigating the stability of the system, and by processing the information acquired through the quantum channel in order to maintain a high security level for the system, and to be able to extract useful information from the raw data. For photon detection, we use single photon avalanche photo diodes (APD's). Their properties in terms of efficiency (probability of detection upon photon arrival) and dark counts (when a detector counts without the presence of a qubit) are highly relevant for information processing. In this chapter we will analyze the most significant sources of errors which are obligatory to govern for above mentioned reasons.

\subsubsection{Rate}

The raw rate $R_{\text {raw }}$ is given by

$$
R_{\text {raw }}=q f \mu \eta_{\text {link }} \epsilon
$$

Eq. $5.4 q=\frac{1}{2}$, due to the fact that half of the time Alice chooses the wrong measurement basis. $f$ is the frequency. $\eta_{l i n k}$ and $\epsilon$ are efficiencies of the quantum channel and single photon detectors, respectively. 
One need to keep in mind that bursting pulses in trains ${ }^{1}$ with master clock frequency $f_{0}$ will give $f=f_{0} \times \frac{\text { Burst }}{\text { loop-time }+ \text { Burst }}$. Loop-time is the time it takes for one pulse to travel from Alice's laser and to the detectors and Burst is the burst length in time. To see how $\eta_{\text {link }}$ is a factor of efficiency, one can look at the ratio between the output and input power. This will leave us the percentage left of the power after passing the link. The output power is of course dependent on the attenuation in the link. If we calculate the difference between the input and output power in units of $\mathrm{dBm}$, we get,

$$
\begin{gathered}
\Delta P=10 \log _{10} \frac{P_{\text {out }}}{P_{\text {in }}} \Rightarrow \frac{P_{\text {out }}}{P_{\text {in }}}=10^{-\Delta P / 10} \text { and } \\
\eta_{\text {link }}=\frac{P_{\text {out }}}{P_{\text {in }}}=10^{-\Delta P / 10}
\end{gathered}
$$

\subsubsection{Visibility}

The whole quantum communication protocol uses an interferometer for measuring and extracting the stored information in a qubit. One need to know how much of the information reaching the interferometer is lost due to the fact that a photon goes to the wrong detector. In other words, the visibility, $\mathrm{V}$, is a measure of how well the interferometric setup works and also how well the assumed theory is related to the experiment. It is defined as eq. $5.6[6]$,

$$
V=\frac{I_{\max }-I_{\min }}{I_{\max }+I_{\min }}
$$

where $I$ denotes intensity, with $I_{\max }$ and $I_{\min }$ being the constructive and destructive interference intensities respectively, on one detector. Using InGaAs APD's, although in gated mode to reduce the dark counts, one will still have a signifact amount of dark counts lowering the visibility, since $I_{\min }$ will have an upper limit due to these dark counts. In order to determine the actual visibility of the interferometer, one has to subtract the dark counts from the APD.s. The visibility equation used in the calculation have the dark counts subtracted.

$$
V=\frac{\left(I_{\max }-I_{\text {dark }}\right)-\left(I_{\min }-I_{\text {dark }}\right)}{\left(I_{\max }-I_{\text {dark }}\right)+\left(I_{\min }-I_{\text {dark }}\right)}=\frac{I_{\max }-I_{\min }}{I_{\max }+I_{\min }-2 I_{\text {dark }}}
$$

Also, the visibility is measured on multi-photon pulses, further separating the errors due to non-perfect extinction ratio in the interferometric system from the dark counts [9].

\footnotetext{
${ }^{1}$ The procedure of bursting pulses in trains will be explained in ch. 6 .
} 


\subsubsection{Quantum Bit Error Rate}

Quantum Bit Error Rate, $Q B E R$, is the amount of bits detected which contain no useful information about the communicated message between parties. $Q B E R$ is given in units of (\%), indicating the amount of the total information detected that should be treated as error bits. The most significant error bits arise from poorly aligned interferometer (or an unstable setup) giving low optical visibilties, $Q B E R_{o p t}$, and dark counts detected in the APD's, $Q B E R_{\text {det }}$. The total error bits are just the sum of contributing factors, $Q B E R=Q B E R_{\text {det }}+Q B E R_{\text {opt }}$. This error have an upper limit of $11 \%$ [15] in order to have a secure quantum communication implementation for BB84-like protocols.

$Q B E R_{o p t}$ is the error due to the optical efficiency of the setup and is hence related to the visibility, $V$ (defined in eq. 5.7), through eq. 5.8 [5],

$$
Q B E R_{\text {opt }}=\frac{1-V}{2}
$$

$Q B E R_{\text {det }}$ is dark count dependent. It is actually the ratio between the probability of detecting a dark count $p_{\text {noise }}$ and the qubit detection probability $p_{\text {photon }}$. The dark count probability is time independent for a detector in constant temperature. However the qubit detection probability is dependent on the attenuation of the entire system. The attenuation of each station provides a constant attenuation of the signal while the attenuation provided by the fiber link between stations increases with distance since standard SMF-28 (single mode fiber) has an attenuation of $\approx 0.25 \mathrm{~dB} / \mathrm{km}$ for $1550 \mathrm{~nm}$ light. The $Q B E R_{\text {det }}$ is also frequency independent since it is the ratio between two probabilities (where they both increase linearly with frequency).

$$
Q B E R_{\text {det }}=\frac{p_{\text {noise }}}{p_{\text {photon }}}=\frac{p_{\text {noise }}}{\mu \epsilon \eta_{\text {link }}}
$$

Eq.5.9 $\epsilon$ is the detectors quantum efficiency, $\eta_{l i n k}$ is the transfer efficiency between the exit point of Bob's station and Alice's detectors ${ }^{2}$.

One can, by knowing the specifications of the components, easily calculate a theoretical plot of the error rate as a function of the distance (fiber link) between Alice and Bob $(L)$. Given a fiber loss of $\approx 0.25 \mathrm{~dB} / \mathrm{km}$, a dark count probability of $1.1 \times 10^{-5}$, a quantum detection efficiency of $20 \%$, a loss in the interferometer of $5 \mathrm{~dB}$ and a mean photon number $\mu=0.1$. One can see, in fig. 5.1, that the maximum theoretical distance is slighlty above $70 \mathrm{~km}$.

\footnotetext{
${ }^{2}$ Notice that Alice is now controlling the detectors instead of Bob. The setup will be explained in detail in ch. 6. It is of course irrelevant regarding the equation.
} 


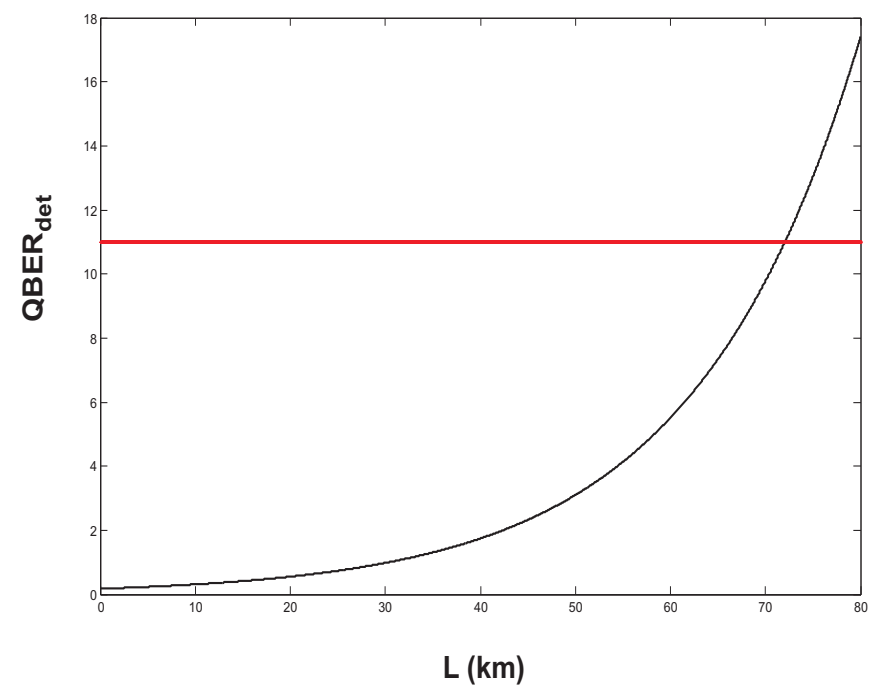

Figure 5.1: Error rate as a function of the distance between users.

One induced avalanche in the APD may give rise to more than one count. This occurs when a carrier is trapped in the multiplier process, and induces a delayed avalanche which the photodiode counts as a photon. This effect is called an afterpulse and is denoted $Q B E R_{a f t e r}$. Each APD have an afterpulse-rate-constant, $\alpha$ (in the order of $10^{-4}$ ), related to this process. The probability of having an afterpulse increases with the frequency (i.e the probability of detecting a photon since the constant is given in units of per detected photon). However, this effect is rather negligible when dealing with frequencies $<5 \mathrm{MHz}$ and attenuations as in our experiments. 


\section{Chapter 6}

\section{Plug and Play Interferometric Setup With Phase Encoding}

\subsection{End-User Plug and Play QKD}

The Plug and Play setup for QKD introduced in 1996 by [2], gives the implementation the advantage of not having the need to be adjusted, calibrated or stabilized more than once. Also, the birefringence problem is solved for light that retraces its path, see sec. 6.1.1 Birefringence. Its purpose is to be ready for a user to turn on his necessary devices and start communicating with other parties, hence the name Plug and Play. The feasibility of an interferometric Plug and Play system using standard telecom fiber on 1550 $\mathrm{nm}$ wavelength has experimentally been shown in [9] [10] [11] [12], reaching distances of up to $67 \mathrm{~km}$ with InGaAs APD's. Distances over $200 \mathrm{~km}$ have also been experimentally reached by using superconducting single photon detectors [13]. There are of course disadvantages as well. For instance, the amount of information flow, Rate $(\mathrm{B} / \mathrm{s})$, in the quantum channel is limited by other measures than the limitations of the electronics, which we also will discuss in due course.

\subsubsection{Birefringence}

Before presenting the setup, we should make it clear why a Faraday mirror is used. Single Mode Fibers (SMF) are unlike polarization maintaining ones birefringent, meaning that the two linear polarization states $|H\rangle$ and $|V\rangle$ propagate at slightly different speeds causing a linear state $|\psi\rangle=$ $\alpha|H\rangle+\beta|V\rangle \rightarrow\left|\psi^{\prime}\right\rangle=\alpha^{\prime}|H\rangle+\beta^{\prime} e^{i \varphi}|V\rangle$, where $\{\alpha, \beta\} \neq\left\{\alpha^{\prime}, \beta^{\prime} e^{i \varphi}\right\}$. 
The $\mathrm{H}$ axis of the fiber is usually called yhe slow axis, and the $\mathrm{V}$ axis is called the fast axis. In other words, while light is propagating through the fiber, its state of polarization, SOP, is influenced by birefringence. A generic retarder $\mathbf{R}$, for this effect can be expressed by eq. 6.1 and 6.2 [3]. The direction of the propagation vector through the fiber is indicated by the arrow index above $\mathbf{R}$. The z-axis, in which the light propagates is chosen as the local direction of propagation so that the light is always traveling in $+z$ direction.

$$
\begin{aligned}
& {[\vec{R}]=\left[\begin{array}{lr}
\cos ^{2} \theta e^{i \varphi / 2}+\sin ^{2} \theta e^{-i \varphi / 2} & i \sin 2 \theta \sin \frac{\varphi}{2} \\
i \sin 2 \theta \sin \frac{\varphi}{2} & \cos ^{2} \theta e^{-i \varphi / 2}+\sin ^{2} \theta e^{i \varphi / 2}
\end{array}\right]} \\
& {[\overleftarrow{R}]=\left[\begin{array}{lr}
\cos ^{2} \theta e^{i \varphi / 2}+\sin ^{2} e^{-i \varphi / 2} & -i \sin 2 \theta \sin \frac{\varphi}{2} \\
-i \sin 2 \theta \sin \frac{\varphi}{2} & \cos ^{2} \theta e^{-i \varphi / 2}+\sin ^{2} e^{i \varphi / 2}
\end{array}\right]}
\end{aligned}
$$

However, there are ways in which one can compensate for this birefringence. It is suggested that a mirrored Faraday rotator is used [3]. The Faraday rotator FR used in our experiment rotates the SOP by exactly $\pi / 4$ per passage, as follows.

$$
\begin{aligned}
& {[\overrightarrow{F R}]=\left[\begin{array}{ll}
\cos \theta & \sin \theta \\
-\sin \theta & \cos \theta
\end{array}\right] \stackrel{\theta=\pi / 4}{\Longrightarrow}[\overrightarrow{F R}]=\frac{1}{\sqrt{2}}\left[\begin{array}{lr}
1 & 1 \\
-1 & 1
\end{array}\right]} \\
& {[\overleftarrow{F R}]=\left[\begin{array}{rr}
\cos \theta & -\sin \theta \\
\sin \theta & \cos \theta
\end{array}\right] \stackrel{\theta=\pi / 4}{\Longrightarrow}[\overleftarrow{F R}]=\frac{1}{\sqrt{2}}\left[\begin{array}{rr}
1 & -1 \\
1 & 1
\end{array}\right]}
\end{aligned}
$$

A Mirrored Faraday Rotator [MFR] can be expressed as eq. 6.5 [4]

$$
\begin{aligned}
{[M F R]=} & {[\overleftarrow{F R}][M][\overrightarrow{F R}]=\frac{1}{2}\left[\begin{array}{rr}
1 & -1 \\
1 & 1
\end{array}\right]\left[\begin{array}{ll}
-1 & 0 \\
0 & 1
\end{array}\right]\left[\begin{array}{ll}
1 & 1 \\
-1 & 1
\end{array}\right]=} \\
& =\frac{1}{2}\left[\begin{array}{rr}
-1 & -1 \\
-1 & 1
\end{array}\right]\left[\begin{array}{ll}
1 & 1 \\
-1 & 1
\end{array}\right]=\left[\begin{array}{lr}
0 & -1 \\
-1 & 0
\end{array}\right]
\end{aligned}
$$

Finally we can compute the operator $[\mathrm{J}]$ for the whole circuit

$$
\begin{gathered}
{[J]=[\overleftarrow{R}][M F R][\vec{R}]=} \\
{\left[\begin{array}{lr}
\cos ^{2} \theta e^{i \varphi / 2}+\sin ^{2} \theta e^{-i \varphi / 2} & -i \sin 2 \theta \sin \frac{\varphi}{2} \\
-i \sin 2 \theta \sin \frac{\varphi}{2} & \cos ^{2} \theta e^{-i \varphi / 2}+\sin ^{2} \theta e^{i \varphi / 2}
\end{array}\right]\left[\begin{array}{lr}
0 & -1 \\
-1 & 0
\end{array}\right] \times}
\end{gathered}
$$




$$
\begin{aligned}
& \times\left[\begin{array}{lr}
\cos ^{2} \theta e^{i \varphi / 2}+\sin ^{2} \theta e^{-i \varphi / 2} & i \sin 2 \theta \sin \frac{\varphi}{2} \\
i \sin 2 \theta \sin \frac{\varphi}{2} & \cos ^{2} \theta e^{-i \varphi / 2}+\sin ^{2} \theta e^{i \varphi / 2}
\end{array}\right]= \\
& {\left[\begin{array}{lr}
i \sin 2 \theta \sin \frac{\varphi}{2} & -\left(\cos ^{2} \theta e^{i \varphi / 2}+\sin ^{2} \theta e^{-i \varphi / 2}\right) \\
-\left(\cos ^{2} \theta e^{-i \varphi / 2}+\sin ^{2} \theta e^{i \varphi / 2}\right) & i \sin 2 \theta \sin \frac{\varphi}{2}
\end{array}\right] \times} \\
& \times\left[\begin{array}{cr}
\cos ^{2} \theta e^{i \varphi / 2}+\sin ^{2} \theta e^{-i \varphi / 2} & i \sin 2 \theta \sin \frac{\varphi}{2} \\
i \sin 2 \theta \sin \frac{\varphi}{2} & \cos ^{2} \theta e^{-i \varphi / 2}+\sin ^{2} \theta e^{i \varphi / 2}
\end{array}\right] \Rightarrow \\
& \left\{\begin{array}{l}
\text { Since } \cos ^{4} \theta+\sin ^{4} \theta+\cos ^{2} \theta \sin ^{2} \theta\left(e^{i \varphi}+e^{-i \varphi}\right)+\sin ^{2} 2 \theta \sin ^{2} \frac{\varphi}{2}= \\
=\cos ^{4} \theta+\sin ^{4} \theta+2 \cos ^{2} \theta \sin ^{2} \theta \cos \left(2 \frac{\varphi}{2}\right)+4 \sin ^{2} \theta \cos ^{2} \theta \sin ^{2} \frac{\varphi}{2}= \\
=\cos ^{4} \theta+\sin ^{4} \theta+2 \cos ^{2} \theta \sin ^{2} \theta\left(\cos \left(2 \frac{\varphi}{2}\right)+2 \sin ^{2} \frac{\varphi}{2}\right)= \\
=\cos ^{4} \theta+\sin ^{4} \theta+2 \cos ^{2} \theta \sin ^{2} \theta\left(\cos ^{2} \frac{\varphi}{2}-\sin ^{2} \frac{\varphi}{2}+2 \sin ^{2} \frac{\varphi}{2}\right)= \\
=\left(\cos ^{2} \theta+\sin ^{2} \theta\right)^{2}= \\
=1
\end{array}\right\} \Rightarrow \\
& \Rightarrow[J]=\left[\begin{array}{lr}
0 & -1 \\
-1 & 0
\end{array}\right]
\end{aligned}
$$

Notice that we have used a 'detector' frame during the calculations above, i.e. as mentioned before, the z-axis in which the light propagates is chosen as the local direction of propagation so that the light is always traveling in $+z$ direction. Basically one can think of it like measuring the SOP after each step, by putting a detector in front of the propagation vector. See example below.

Let $|\psi\rangle=\alpha|H\rangle+\beta|V\rangle$. This is shown by the following example (leaving out the unit vectors $\bar{H}$ and $\bar{V}$ ). See eq. 6.7 and fig. 6.1.

$$
\left|\psi^{\prime}\right\rangle=[J]|\psi\rangle=\left[\begin{array}{lr}
0 & -1 \\
-1 & 0
\end{array}\right]\left[\begin{array}{l}
\alpha \\
\beta
\end{array}\right]=\left[\begin{array}{l}
-\beta \\
-\alpha
\end{array}\right]
$$



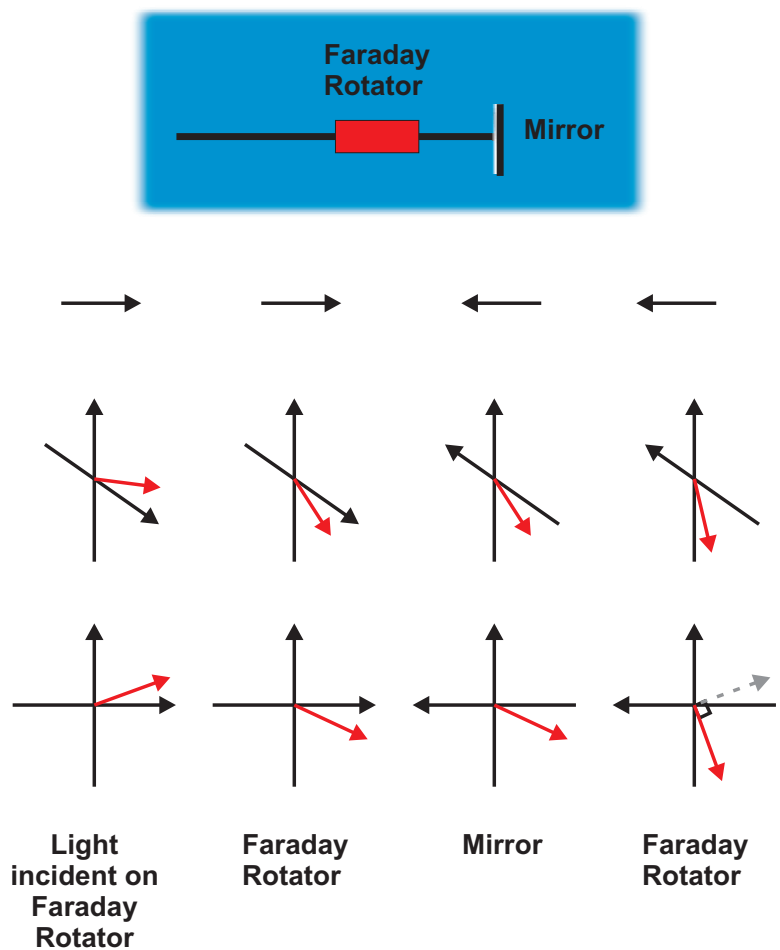

Faraday

Mirror

Faraday

Rotator

Figure 6.1: Scheme for Faraday rotation

In our case, Alice sends out two linear polarized vectors from her PBS which has a fix axis, unlike a 'detector' frame. Consider fig. 6.1 without the change of the axis $\bar{H} \rightarrow-\bar{H}$, i.e. the light first travels in $+z$ direction and returns in $-z$ direction. If we were to mirror back the $-\bar{H}$, eq. 6.6 becomes

$$
\left[J^{\prime}\right]=[M][J]=\left[\begin{array}{ll}
-1 & 0 \\
0 & 1
\end{array}\right]\left[\begin{array}{lr}
0 & -1 \\
-1 & 0
\end{array}\right]=\left[\begin{array}{ll}
0 & 1 \\
-1 & 0
\end{array}\right]
$$

So according to Alice's PBS, the state is

$$
\left|\psi^{\prime}\right\rangle=\left[J^{\prime}\right]|\psi\rangle=\left[\begin{array}{ll}
0 & 1 \\
-1 & 0
\end{array}\right]\left[\begin{array}{l}
\alpha \\
\beta
\end{array}\right]=\left[\begin{array}{l}
\beta \\
-\alpha
\end{array}\right]
$$

Or, in a more general case

$$
\begin{gathered}
\left|\psi^{\prime}\right\rangle=\left[J^{\prime}\right]|\psi\rangle=\left[\begin{array}{ll}
0 & 1 \\
-1 & 0
\end{array}\right]\left[\begin{array}{l}
\alpha \\
\beta e^{i \varphi}
\end{array}\right]=\left[\begin{array}{l}
\beta e^{i \varphi} \\
-\alpha
\end{array}\right] \Rightarrow \\
\Rightarrow\left\langle\psi^{\prime} \mid \psi\right\rangle=\alpha \beta\left(e^{i \varphi}-e^{-i \varphi}\right)=0 \text {, if } \varphi=\pi k, k \in Z \text { and } \alpha, \beta \in R \backslash 0 .
\end{gathered}
$$

This means that when input light is linearly polarized, then a linear polarized light is recovered, orthogonal to the input light. But notice that 
the PBS is not experiencing the fact that, $|\psi\rangle$ and $\left|\psi^{\prime}\right\rangle$ have different signs on their propagation vectors. Now, since Alice sends strictly $|H\rangle$ and strictly $|V\rangle$ through her PBS, $|V\rangle$ and $|H\rangle$ is recovered respectively, regardless of which frame one wishes to apply the operator matrix. They both deduce to the same outcome, namely:

$$
|\psi\rangle=\alpha|H\rangle \Rightarrow\left\{\begin{array}{l}
\left|\psi^{\prime}\right\rangle=[J]|\psi\rangle=\left[\begin{array}{lr}
0 & -1 \\
-1 & 0
\end{array}\right]\left[\begin{array}{l}
\alpha \\
0
\end{array}\right]=\left[\begin{array}{l}
0 \\
-\alpha
\end{array}\right] \equiv \alpha|V\rangle \\
\left|\psi^{\prime}\right\rangle=\left[J^{\prime}\right]|\psi\rangle=\left[\begin{array}{ll}
0 & 1 \\
-1 & 0
\end{array}\right]\left[\begin{array}{l}
\alpha \\
0
\end{array}\right]=\left[\begin{array}{l}
0 \\
-\alpha
\end{array}\right] \equiv \alpha|V\rangle
\end{array}\right.
$$

and,

$$
|\psi\rangle=\alpha|V\rangle \Rightarrow\left\{\begin{array}{l}
\left|\psi^{\prime}\right\rangle=[J]|\psi\rangle=\left[\begin{array}{lr}
0 & -1 \\
-1 & 0
\end{array}\right]\left[\begin{array}{l}
0 \\
\alpha
\end{array}\right]=\left[\begin{array}{l}
-\alpha \\
0
\end{array}\right] \equiv \alpha|H\rangle \\
\left|\psi^{\prime}\right\rangle=\left[J^{\prime}\right]|\psi\rangle=\left[\begin{array}{lr}
0 & 1 \\
-1 & 0
\end{array}\right]\left[\begin{array}{l}
0 \\
\alpha
\end{array}\right]=\left[\begin{array}{l}
\alpha \\
0
\end{array}\right] \equiv \alpha|H\rangle
\end{array}\right.
$$

In both eq. 6.9 and 6.10 , we get:

$$
\left\langle\psi \mid \psi^{\prime}\right\rangle=0
$$

\subsubsection{Setup}

The Plug and Play setup has the major advantage of stability. There is no need for users to calibrate interferometers or other components in the same manner as a one-way Mach-Zehnder interferometer, which is sensitive to (even very slow) vibrations and temperature fluctuations, making the path difference dependent on these variables. Fig. 6.2 shows an optical fiber version of a Mach-Zehnder interferometer without the presence of any phase modulators. The interference of the two split pulses is now only depending on the optical path difference, $\Delta L$. However, the smallest mechanical stress, $\tau$, on the fiber or any temperature fluctuations (assume that the two arms are influenced by different temperature fluctuations), $T$, will contribute to fluctuations in the interference intensity, $I_{i}$, i.e the intensity will not be stable. Eq. 2.1 will here instead become eq. 6.12. 


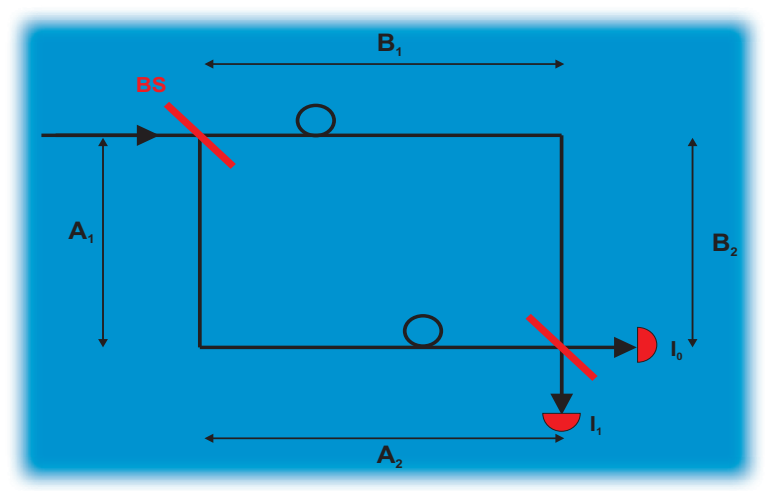

Figure 6.2: One way optical fiber Mach-Zehnder interferometer.

$$
\left\{\begin{array}{l}
I_{0}(\Delta L)=I_{s} \cos ^{2}\left(\frac{k \Delta L}{2}\right) \\
\Delta L=\left(A_{1}+A_{2}\right)-\left(B_{1}+B_{2}\right)
\end{array}\right.
$$

And since,

$$
\begin{gathered}
A_{i}=A_{i}(T, \tau), B_{i}=B_{i}(T, \tau) \Rightarrow \Delta L=\Delta L(T, \tau) \Rightarrow \\
\Rightarrow I_{0}(\Delta L)=I_{0}(T, \tau)
\end{gathered}
$$

Before introducing the Plug and Play setup which provide a solution for this problem, let us first analyze a polarization beam splitter, (PBS), used in the setup. The PBS has three I/O ports T, 1 and 2 (where T stands for common). Port 1 and 2 are aligned to the slow axis (i.e. horizontal polarized light) and have a very high attenuation for vertical polarized light. A figure of a PBS and a scheme for the same are shown in fig. 6.3 and table 6.1, respectively.

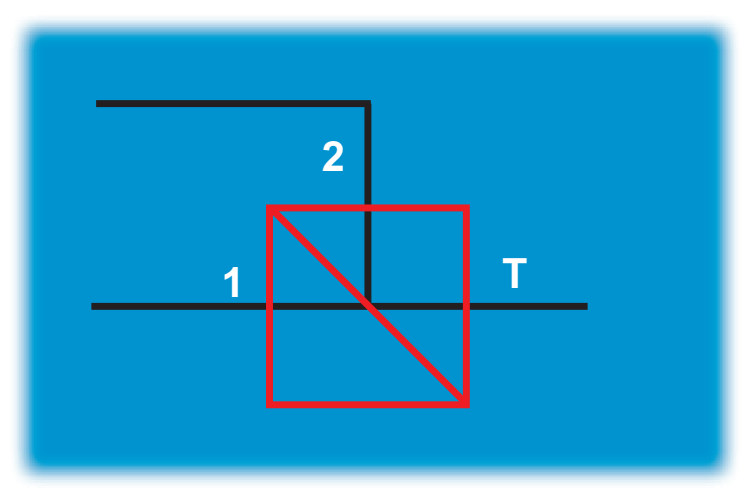

Figure 6.3: Polarization beam splitter. 


\begin{tabular}{cccc}
\hline \hline Input Port & Input Polarization & Output Port & Output Polarization \\
$\mathrm{T}$ & $|H\rangle$ & 1 & $|H\rangle$ \\
$\mathrm{T}$ & $|V\rangle$ & 2 & $|H\rangle$ \\
1 & $|H\rangle$ & $\mathrm{T}$ & $|H\rangle$ \\
2 & $|H\rangle$ & $\mathrm{T}$ & $|V\rangle$ \\
\hline
\end{tabular}

Table 6.1: Scheme for a polarization beam splitter.

Now, let us analyze what will happen if we were to put a Faraday mirror after port T. A figure of a PBS attached to a Faraday mirror and the scheme for the same are shown in fig. 6.4 and table 6.2.

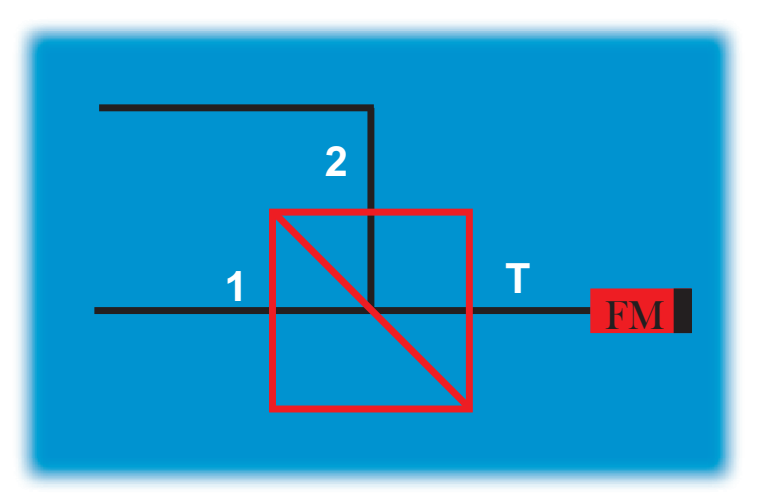

Figure 6.4: Polarization Beam Splitter attached to a Faraday Mirror

\begin{tabular}{cccc}
\hline \hline Input & Output Pol. & Input Pol. (Port T, & Output \\
Port $(|H\rangle)$ & (Port T) & after FM reflection) & Port $(|H\rangle)$ \\
1 & $|H\rangle$ & $|V\rangle$ & 2 \\
2 & $|V\rangle$ & $|H\rangle$ & 1 \\
\hline
\end{tabular}

Table 6.2: Scheme for a polarization beam splitter attached to a Faraday Mirror.

\section{Alice, The Interferometer}

We are now ready to analyze the optical fiber version of the Mach-Zehnder interferometer we used in our setup. Consider fig. 6.5 and notice that all the components are aligned to the horizontal axis and have high attenuation for the vertical one. A pulse is sent from the laser diode to the interferometer input after passing a polarizer, where only horizontal polarized light is let to transmit. Once the pulse reaches inside the interferometers it passes an optical circulator $\mathrm{C}$. It is then split through a standard $2 \mathrm{x} 2$ beam splitter (BS), into two arms, where the upper arm is called the short arm and the 
lower arm is called the long arm. The pulse traveling in the long arm passes a phase modulator (but no modulation takes place) and is trapped in a 50 $\mathrm{ns}(10 \mathrm{~m})$ delay line. The purpose of this delay line is to give other parties a chance to modulate one pulse without perturbing the other. The short arm pulse passes the PBS and is reflected by the Faraday mirror and is then sent into the long arm (as discussed in the PBS analysis above). Once the long arm pulse escapes the delay line, it passes the PBS and is reflected by the Faraday mirror into the short arm. Now, when the short arm pulse (currently reflected into the long arm) reaches the phase modulator, we are able to modulate its phase i.e. phase shift it with a $\phi_{A}$, relative to its twin pulse. Since the two pulses have traveled the exact same optical path (i.e $\Delta L=0$ ), it is obvious that they will interfere with each other at the beam splitter and that the interference will solely depend on the phase modulation.

Notice that any temperature fluctuations (even if different on the two arms) or mechanical stress on the fiber in one of the arms will not contribute to any interference disturbance, as long as this perturbation varies slower than the time it takes for the two pulses to travel across the perturbation segment, since they will then be perturbed in the same manner. One is able to achieve great stability and visibility through this setup. So our interference intensity equations now become,

$$
\begin{aligned}
& I_{1}=I \cos ^{2}\left(\frac{\phi_{A}}{2}\right) \\
& I_{0}=I \sin ^{2}\left(\frac{\phi_{A}}{2}\right)
\end{aligned}
$$

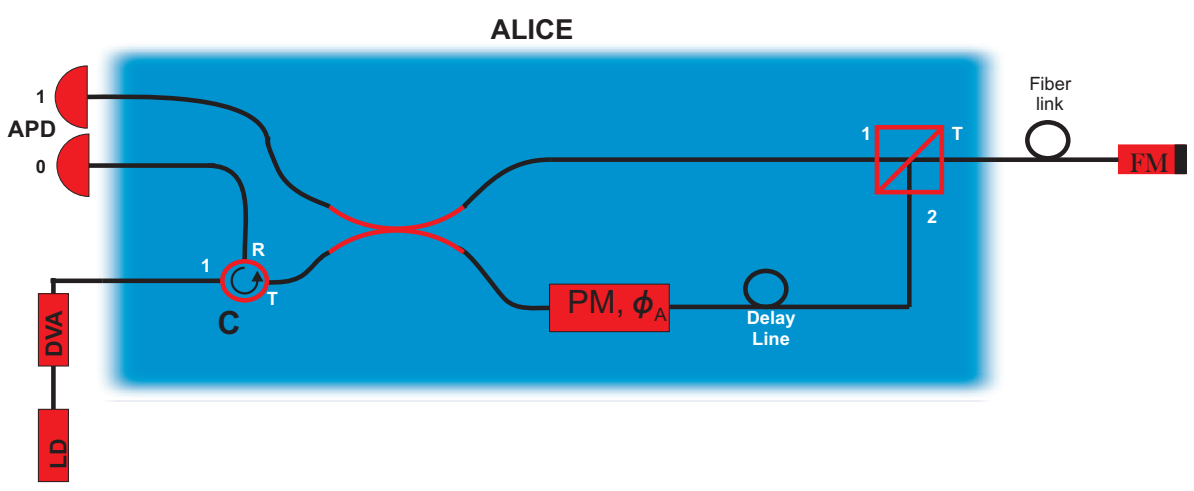

Figure 6.5: Alice - the interferometer. All components and interconnecting fiber inside the interferometer are polarization maintaining fiber. DVA, digital variable attenuator. We should also mention that the transmission scheme for circulator $\mathrm{C}$ is, $1 \rightarrow \mathrm{T}$ and $\mathrm{T} \rightarrow \mathrm{R}$

Let us analyze the interference procedure step by step. The laser diode sends a pulse (or qubit) in an initial state $\left|\psi_{0}\right\rangle$ at time $t_{0}$. The first entry in the kets ${ }^{1}$ (below) denotes the time part of the vector, whereas the

\footnotetext{
${ }^{1}\langle x|$ is called a bra and $|x\rangle$ is called a ket. $x$ is the entry.
} 
following entries keep a historical track of the spatial part of the vector with the last spatial entry denoting the present position. $U$ denotes upper arm and $L$ denotes lower arm. During this derivation, we will keep any normalization constants out since they will not affect the desired outcome of the derivation. To justify this claim, it should be mentioned that it is of utmost importance that all components have symmetrical attenuation, meaning that light traveling clockwise will experience the same attenuation as light traveling counterclockwise. To achieve this in the experimental part of the thesis, the attenuation of all components have carefully been measured and given specific locations in the setup. Alice's phase modulator have for instance near perfect symmetrical attenuation, one of very few phase modulators at hand have this property. Non-symmetrical attenuating components will give rise to lower optical visibility, since intensity is a factor of relevance for interference. It should be mentioned that a transmitted and a reflected pulse through a $2 \times 2$ beam splitter experience a $\pi / 2$ relative phase shift. This basically means that a pulse which is split into the two arms of the interferometer will have a relative phase shift. This can also be seen in the derivation below from the first arrow indicator. Table 6.3 is to be used as a guide for the followed derivation.

\begin{tabular}{cl}
\hline \hline Arrow superscript & Component \\
\hline 1 & 2x2 Beam Splitter \\
2 & Delay Line \\
3 & Polarization Beam Splitter \\
4 & Faraday Mirror \\
5 & Polarization Beam Splitter \\
6 & Delay Line \\
7 & Phase Modulator \\
8 & 2x2 Beam Splitter \\
\hline
\end{tabular}

Table 6.3: Reference table for derivation below 


$$
\begin{aligned}
& \left|\psi_{0}\right\rangle=\left|t_{0}\right\rangle_{H} \stackrel{1}{\rightarrow} \\
& \left|t_{1}, U\right\rangle_{H}+e^{i \pi / 2}\left|t_{1}, L\right\rangle_{H} \stackrel{2}{\rightarrow} \\
& \left|t_{2}, U\right\rangle_{H}+e^{i \pi / 2}\left|t_{2}+t_{d l}, L\right\rangle_{H} \stackrel{3}{\rightarrow} \\
& \left|t_{3}, U\right\rangle_{H}+e^{i \pi / 2}\left|t_{3}+t_{d l}, L\right\rangle_{V} \stackrel{4}{\rightarrow} \\
& -\left|t_{4}, U\right\rangle_{V}+e^{i \pi / 2}\left|t_{4}+t_{d l}, L\right\rangle_{H} \stackrel{5}{\rightarrow} \\
& -\left|t_{5}, U, L\right\rangle_{H}+e^{i \pi / 2}\left|t_{5}+t_{d l}, L, U\right\rangle_{H} \stackrel{6}{\rightarrow} \\
& -\left|t_{6}+t_{d l}, U, L\right\rangle_{H}+e^{i \pi / 2}\left|t_{6}+t_{d l}, L, U\right\rangle_{H} \stackrel{7}{\rightarrow} \\
& -e^{i \phi_{A}}\left|t_{7}+t_{d l}, U, L\right\rangle_{H}+e^{i \pi / 2}\left|t_{7}+t_{d l}, L, U\right\rangle_{H} \stackrel{8}{\rightarrow} \\
& \left|\psi^{\prime}\right\rangle=-e^{i \phi_{A}}\left(\left|t_{8}, U, L, U\right\rangle_{H}+e^{i \pi / 2}\left|t_{8}, U, L, L\right\rangle_{H}\right)+ \\
& +e^{i \pi / 2}\left(e^{i \pi / 2}\left|t_{8}, L, U, U\right\rangle_{H}+\left|t_{8}, L, U, L\right\rangle_{H}\right)
\end{aligned}
$$

Now, all the vectors are coincident in the same time frame, i.e. all timebin vectors are correlated in time. We need to analyze their space properties. We see that $\left|t_{8}, U, L, U\right\rangle_{H}$ interfere with $\left|t_{8}, L, U, U\right\rangle_{H}$ and $\left|t_{8}, U, L, L\right\rangle_{H}$ interfere with $\left|t_{8}, L, U, L\right\rangle_{H}$, since the last entry in the brackets indicates the current spatial position of the vector. First of all, let us clean up the vectors by defining our final state $\left|\psi^{\prime}\right\rangle$, and introducing the normalization factors.

$$
\left\{\begin{array}{l}
\left|\psi_{0}\right\rangle \rightarrow\left|\psi^{\prime}\right\rangle=\left|\psi_{U}\right\rangle+\left|\psi_{L}\right\rangle \\
\left|\psi_{U}\right\rangle=\frac{1}{2}\left(\left|U_{1}\right\rangle+\left|U_{2}\right\rangle\right) \\
\left|\psi_{L}\right\rangle=\frac{1}{2}\left(\left|L_{1}\right\rangle+\left|L_{2}\right\rangle\right) \\
\left|U_{1}\right\rangle=-e^{i \phi_{A}}\left|t_{8}, U, L, U\right\rangle_{H} \\
\left|U_{2}\right\rangle=e^{i \pi}\left|t_{8}, L, U, U\right\rangle_{H} \\
\left|L_{1}\right\rangle=-e^{i\left(\pi / 2+\phi_{A}\right)}|t, U, L, L\rangle_{H} \\
\left|L_{2}\right\rangle=e^{i \pi / 2}|t, L, U, L\rangle_{H} \\
\left|\psi_{U}\right|^{2}=\left\langle\psi_{U} \mid \psi_{U}\right\rangle=\frac{1}{4}\left(\left\langleU _ { 1 } | + \langle U _ { 2 } | ) \left(\left|U_{1}\right\rangle+\left|U_{2}\right\rangle=\right.\right.\right.
\end{array}\right.
$$$$
\frac{1}{4}\left(1+1+2 \cos \left(\phi_{A}+\pi-\pi\right)\right)=\frac{1}{2}\left(1+\cos \left(\phi_{A}\right)\right) \stackrel{\text { def. }}{=} \cos ^{2}\left(\frac{\phi_{A}}{2}\right)
$$ 


$$
\begin{aligned}
& \left|\psi_{L}\right|^{2}=\left\langle\psi_{L} \mid \psi_{L}\right\rangle=\frac{1}{4}\left(\left\langleL _ { 1 } | + \langle L _ { 2 } | ) \left(\left|L_{1}\right\rangle+\left|L_{2}\right\rangle=\frac{1}{4}\left(1+1+2 \cos \left(\phi_{A}+\pi\right)=\right.\right.\right.\right. \\
& \frac{1}{2}\left(1+\cos \left(\phi_{A}+\pi\right)\right) \stackrel{\text { def. }}{=} \cos ^{2}\left(\frac{\phi_{A}+\pi}{2}\right)=\sin ^{2}\left(\frac{\phi_{A}}{2}\right)
\end{aligned}
$$

The two arms connected to the APD's through the beam splitter have a phase difference of $\pi$, which is exactly what eq. 6.13 is indicating, i.e. as Alice changes the phase modulation from $0 \rightarrow \pi$, the constructive interference completely changes arm. As mentioned previously, when dealing with quantum states, the expressions in eq. 6.13 become probabilities instead, where $P\left(I_{i}\right)$ is the probability that the photon is detected by the APD number $i$. A plot of expressions in eq. 6.14 is shown in fig. 6.6.

$$
\begin{aligned}
& P\left(I_{1}\right)=\cos ^{2}\left(\frac{\phi_{A}}{2}\right) \\
& P\left(I_{0}\right)=\sin ^{2}\left(\frac{\phi_{A}}{2}\right)
\end{aligned}
$$

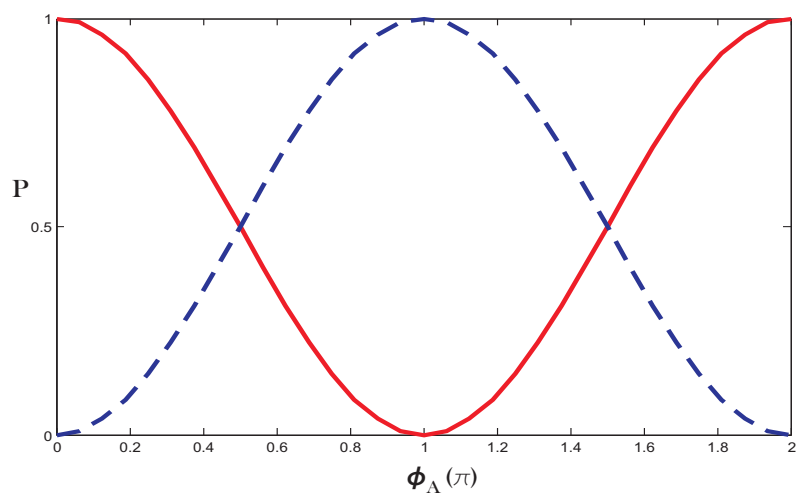

Figure 6.6: Plot of expressions in eq. 6.14

\section{Bob}

We can now add a user, Bob, who is able to phase modulate one of the pulses. The ideal case were to design Bob as in fig. 6.7. However, since the available phase modulators are aligned to the slow axis i.e. horizontal polarized light, it strongly attenuates vertical polarized light, i.e the phase modulators are polarization sensitive. Also, it is hard to predict the polarization after transmission through the fiber. So we had to design Bob's station in such a way so that the polarization sensitive phase modulators could serve their purpose without disturbing or filtering out a polarization state which is incompatible with it. Fig. 6.7 is showing Bob's setup, if we were to have a polarization insensitive phase modulator. However, since they are not available, we had to design Bob's station as shown in fig. 6.8. 


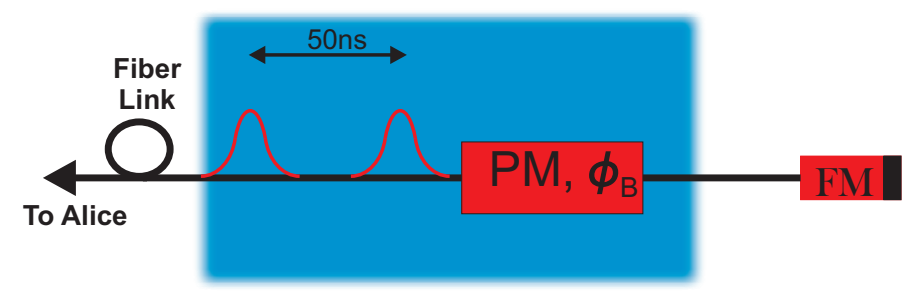

Figure 6.7: The ideal setup for Bob, in the case with a polarization insensitive phase modulator.

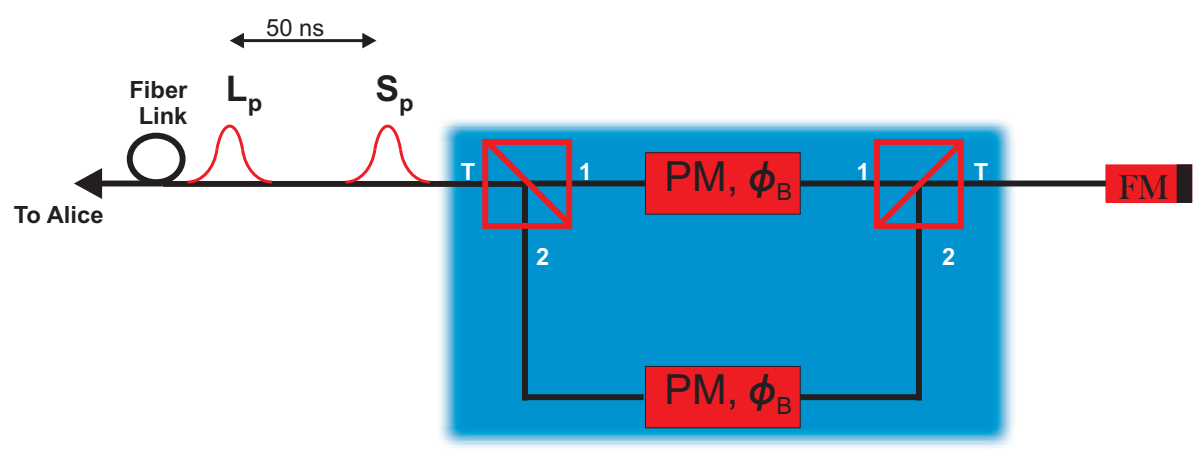

Figure 6.8: Bob's setup, using two polarization sensitive phase modulators.

Following the same procedure as with the analysis of Alice's interferometer, we analyze Bob's setup. The short arm pulse $S_{p}$ arrive approx. $50 \mathrm{~ns}$ before the long arm pulse $L_{p}$, since $L_{p}$ has traveled through Alice's phase modulator and also the delay line. $S_{p}$ is split up according to its SOP into Bob's two arms, upper $U$ and lower $L$. The two parts of $S_{p}$ are phase modulated by Bob as they are on the way to the Faraday mirror. They are thereafter reflected and sent back to Alice, while $L_{p}$ is still traveling in the fiber link. Once $L_{p}$ enters Bob's station, he will keep his phase modulation off, and let $L_{p}$ be reflected by the Faraday mirror and sent back to Alice. An analysis of the short arm pulse is done below. Again we mention that table 6.4 serves as a reference table for the followed derivation. A similar analysis apply for the long arm pulse. 


\begin{tabular}{cl}
\hline \hline Arrow superscript & Component \\
\hline 1 & PBS (left) \\
2 & Phase modulator \\
3 & PBS (right) \\
4 & Farady Mirror \\
5 & PBS (right) \\
6 & PBS (left) \\
7 & Fiber link \\
\hline
\end{tabular}

Table 6.4: Reference table for derivation below

$$
\begin{aligned}
& \left|S_{p}\right\rangle=\alpha\left|s_{p}\right\rangle_{H}+\beta e^{i \varphi}\left|s_{p}\right\rangle_{V} \stackrel{1}{\rightarrow} \\
& \alpha\left|s_{p}, U\right\rangle_{H}+\beta e^{i \varphi}\left|s_{p}, L\right\rangle_{H} \stackrel{2}{\rightarrow} \\
& \alpha e^{i \phi_{B}}\left|s_{p}, U\right\rangle_{H}+\beta e^{i\left(\varphi+\phi_{B}\right)}\left|s_{p}, L\right\rangle_{H} \stackrel{3}{\rightarrow} \\
& \alpha e^{i \phi_{B}}\left|s_{p}, U\right\rangle_{H}+\beta e^{i\left(\varphi+\phi_{B}\right)}\left|s_{p}, L\right\rangle_{V} \stackrel{4}{\rightarrow} \\
& -\alpha e^{i \phi_{B}}\left|s_{p}, U\right\rangle_{V}+\beta e^{i\left(\varphi+\phi_{B}\right)}\left|s_{p}, L\right\rangle_{H} \stackrel{5}{\rightarrow} \\
& -\alpha e^{i \phi_{B}}\left|s_{p}, U, L\right\rangle_{H}+\beta e^{i\left(\varphi+\phi_{B}\right)}\left|s_{p}, L, U\right\rangle_{H} \stackrel{6}{\rightarrow} \\
& -\alpha e^{i \phi_{B}}\left|s_{p}, U, L\right\rangle_{V}+\beta e^{i\left(\varphi+\phi_{B}\right)}\left|s_{p}, L, U\right\rangle_{H} \stackrel{7}{\rightarrow} \\
& e^{i \phi_{B}}\left(-\alpha\left|s_{p}, U, L, F L\right\rangle_{V}+\beta e^{i \varphi}\left|s_{p}, L, U, F L\right\rangle_{H}\right)= \\
& =e^{i \phi_{B}}\left|S_{p^{\prime}}\right\rangle
\end{aligned}
$$

Eq. 6.15. The last entry in brackets, FL, denotes fiber link back to Alice, to make it clear that they are now coupled.

Any birefringence or change of the SOP introduced by Bob's setup is to be disregarded, since possible perturbation by his setup falls under the derivation we did with a generic retarder $\mathbf{R}$, discussed in 6.1.1 Birefringence. This means that, even in Bob's station, a linear polarized light input will return orthogonal to the input light. This can easily be seen by putting $\varphi=0$ in eq. 6.15 and see that $\left\langle S_{p} \mid S_{p^{\prime}}\right\rangle=0$. Also, the phase modulators of Bob have carefully been selected in order to attenuate the two linear vectors traveling clockwise and counterclockwise in the same manner. 


\section{Storage Line and Attenuator}

Before we view the entire setup of the Plug and Play QKD, we need to introduce some other components that are necessary for the setup. First of all, we need to introduce a so called storage line to Bob's setup, for two main reasons. The first reason is due to Rayleigh backscattering. Rayleigh backscattering is caused by the inhomogeneities in the refractive index of the fiber and means that when the strong classical pulse is traveling from Alice to Bob, some backscattered photons are trapped in the spatial fiber mode and propagates in the backward direction. Since the backscattered light is of the same wavelength as the initial pulse, it cannot be decoupled using optical filters and will end up in the detectors which are sensitive to that exact wavelength. Furthermore, since this backscattering can take place at any time, it cannot either be avoided by using certain gating times for the detectors. So, in [14] it is suggested to use a storage line located at Bob's station. Alice then sends a train of pulses to Bob, filling the storage line, and will not send any new train until all of the pulses have returned. Since she knows when this train is coming back, she only gates the detectors during this period and stops the gating process while sending a new train to Bob. This way, backscattering rate detected by Alice will drop significantly.

The second reason for pulsing the laser in trains (bursts) into the storage line is to avoid an overlap in Alice's phase modulator between incoming qubits, which are being phase modulated, and any outgoing classical pulse she is sending to Bob. The storage line can easily be put on the fiber link side, i.e to the right side of Bob's station in fig. 6.9, when the pulsing period is lower than the time it takes for the short arm and the long arm pulse to enter and exit Bob's station. Otherwise, it is wise to put it in front of the Faraday mirror, letting Bob phase modulate pulses on the way back from the Faraday mirror. It should also be noticed that Alice is now the sender of the classical pulsed light but the receiver of qubits. This means that Alice sends a strong classical pulse to Bob. However when the pulse is leaving Bob's station, it is on single photon level. Now, we want to make sure that the communication at quantum level starts on Bob's side, making him the qubit sender. We could attach a digital variable attenuator (DVA) to his setup, allowing him to attenuate the signal to a single photon level. But then again, there would be some issues with a backscattering effect, although not of the type of Rayleigh backscattering. Since the attenuator simply blocks some part of the beam, there will be backscattered light. Instead, we put the attenuator on Alice's station. Alice then attenuates the pulses in such a way that they exit Bob's station on single photon level. It is obvious that these parameters, which force us to send pulses in trains into the storage line, limits the information rate $(\mathrm{B} / \mathrm{s})$ communicated between Alice and Bob, compared to continuous pulsed light. 


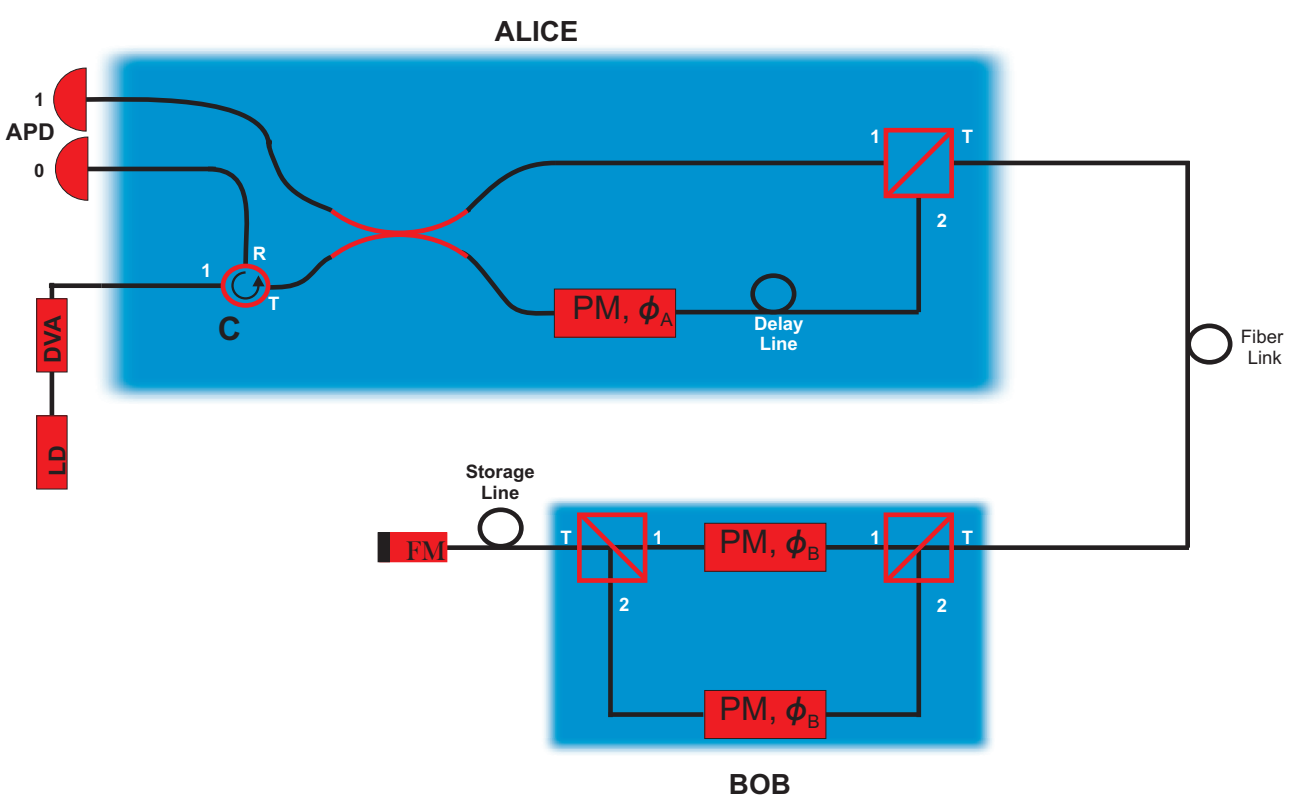

Figure 6.9: Setup of Plug and Play QKD.

\subsection{Multi-User Plug and Play QKD Network}

Here we present the Plug and Play setup for our Multi-User QKD implementation in detail. A detailed version of the tree configuration can be seen in fig. 6.10, (notice that all end users have a storage line in the exact same way as shown in Elisabeth's station). The detailed star configuration is not necessary to view since all principles are identical. Actually, the only thing that needs a thorough clarification is the deviant Elisabeth. As one can see, she has only one phase modulator. This is due to the fact that one, and only one phase modulator is sufficient, even when regarding the standard 2-user phase encoding QKD implementation. An important property required is that the phase modulator has symmetrical attenuation. Also, the position of Elisabeth's storage line is very important, since dealing with one phase modulator. Other stations may place the storage line in front of the Faraday mirror. We will analyze Elisabeth's station in the same manner as we did for Alice's interferometer and Bob's station. As the short arm pulse reaches her station, the photon will split up into the the two arms, upper $U$ and lower $L$. While the lower part reaches the phase modulator we apply a phase shift pulse. We keep this phase shift pulse until the upper pulse is reflected into the lower arm and is also phase shifted. Now, this procedure must take place during less than $50 \mathrm{~ns}$ time, since thereafter the long arm pulse (of Alice) will enter Elisabeth's station. This is why it is not wise to place Elisabeth's storage line in front of the Faraday mirror. Table 6.5 serves as a reference 
table for the followed derivation.

\begin{tabular}{cl}
\hline \hline Arrow superscript & Component \\
\hline 1 & PBS (left) \\
2 & Phase modulator \\
3 & PBS (right) \\
4 & Farady Mirror \\
5 & PBS (right) \\
6 & Phase Modulator \\
7 & PBS (left) \\
8 & Fiber link \\
\hline
\end{tabular}

Table 6.5: Reference table for derivation below. Regarding Elisabeth's station

$$
\begin{gathered}
\left|S_{p}\right\rangle=\alpha\left|s_{p}\right\rangle_{H}+\beta e^{i \varphi}\left|s_{p}\right\rangle_{V} \stackrel{1}{\rightarrow} \alpha\left|s_{p}, U\right\rangle_{H}+\beta e^{i \varphi}\left|s_{p}, L\right\rangle_{H} \stackrel{2}{\rightarrow} \\
\alpha\left|s_{p}, U\right\rangle_{H}+\beta e^{i\left(\varphi+\phi_{E}\right)}\left|s_{p}, L\right\rangle_{H} \stackrel{3}{\rightarrow} \alpha\left|s_{p}, U\right\rangle_{H}+\beta e^{i\left(\varphi+\phi_{E}\right)}\left|s_{p}, L\right\rangle_{V} \stackrel{4}{\rightarrow} \\
-\alpha\left|s_{p}, U\right\rangle_{V}+\beta e^{i\left(\varphi+\phi_{E}\right)}\left|s_{p}, L\right\rangle_{H} \stackrel{5}{\rightarrow} \\
-\alpha\left|s_{p}, U, L\right\rangle_{H}+\beta e^{i\left(\varphi+\phi_{E}\right)}\left|s_{p}, L, U\right\rangle_{H} \stackrel{6}{\rightarrow} \\
-\alpha e^{i \phi_{E}}\left|s_{p}, U, L\right\rangle_{H}+\beta e^{i\left(\varphi+\phi_{E}\right)}\left|s_{p}, L, U\right\rangle_{H} \stackrel{7}{\rightarrow} \\
-\alpha e^{i \phi_{E}}\left|s_{p}, U, L\right\rangle_{V}+\beta e^{i\left(\varphi+\phi_{E}\right)}\left|s_{p}, L, U\right\rangle_{H} \stackrel{8}{\rightarrow} \\
e^{i \phi_{E}}\left(-\alpha\left|s_{p}, U, L, F L\right\rangle_{V}+\beta e^{i \varphi}\left|s_{p}, L, U, F L\right\rangle_{H}\right)= \\
=e^{i \phi_{E}}\left|S_{p^{\prime}}\right\rangle
\end{gathered}
$$

Eq. 6.16. The last entry in brackets, FL, denotes fiber link back to Alice. This is to make it clear that they are now coupled

Again, we emphasize that end-users' stations are to be regarded somewhat of a birefringence effect, which is compensated for due to the Faraday mirror reflection. Also, notice that eq. 6.16 is identical to eq. 6.15 which included two phase modulators. The detailed tree-configuration is shown in fig. 6.10. The star-like configuration is not shown in detail, since it is quite similar. 


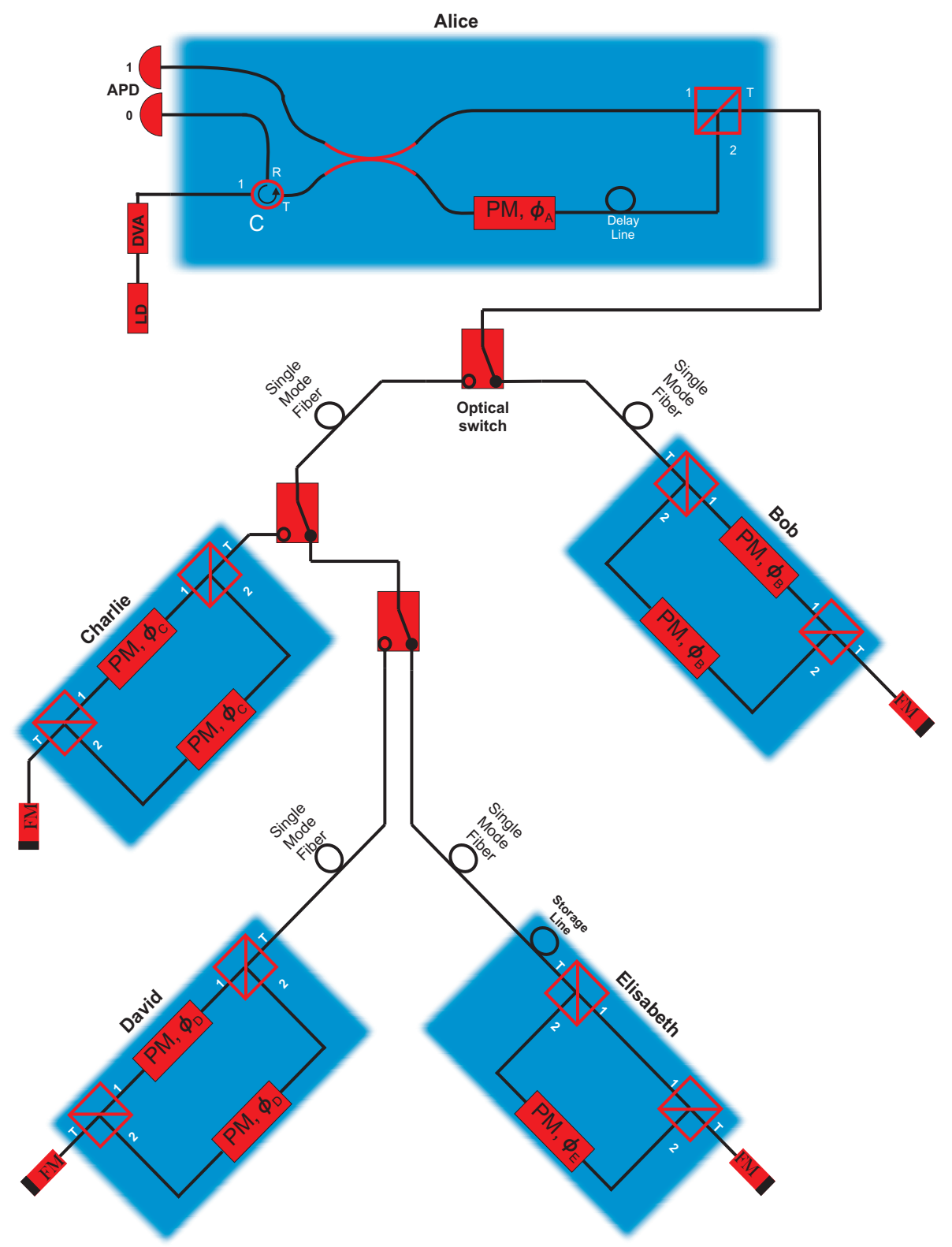

Figure 6.10: A detailed figure of the tree configuration. Notice that all users have a storage line as in Elisabeth's station.

\subsection{Plug and Play Quantum Secret Sharing}

We have implemented a Plug and Play setup for the Secret Sharing protocol, since previous reasons for using a Plug and Play setup are still valid. Recall our discussion for a 2-end-user Plug and Play QKD setup. We will now add 3 more parties (in series) to that setup, as it is shown in fig. 6.11. Elisabeth's 
station works here in the same manner as discussed in the Multi-user QKD implementation. A pulse leaves Alice's station and is split into two arms. As the pulse is traveling toward Elisabeth, no phase modulation takes place by any party. The two parts of the pulse will enter the fiber link, with the short arm pulse traveling $\approx 50$ ns before the long arm pulse. Now, once the pulses are reflected by the Faraday mirror, the qubit encoding starts with Elisabeth. She phase modulates one of the pulses (say, the short arm pulse) and leave the long arm pulse unaltered as a reference pulse. The same apply for all parties. Once the qubit enter Alice's station, the short arm pulse will be transmitted into the long arm, giving Alice the opportunity to perform the final phase modulation: setting a base.

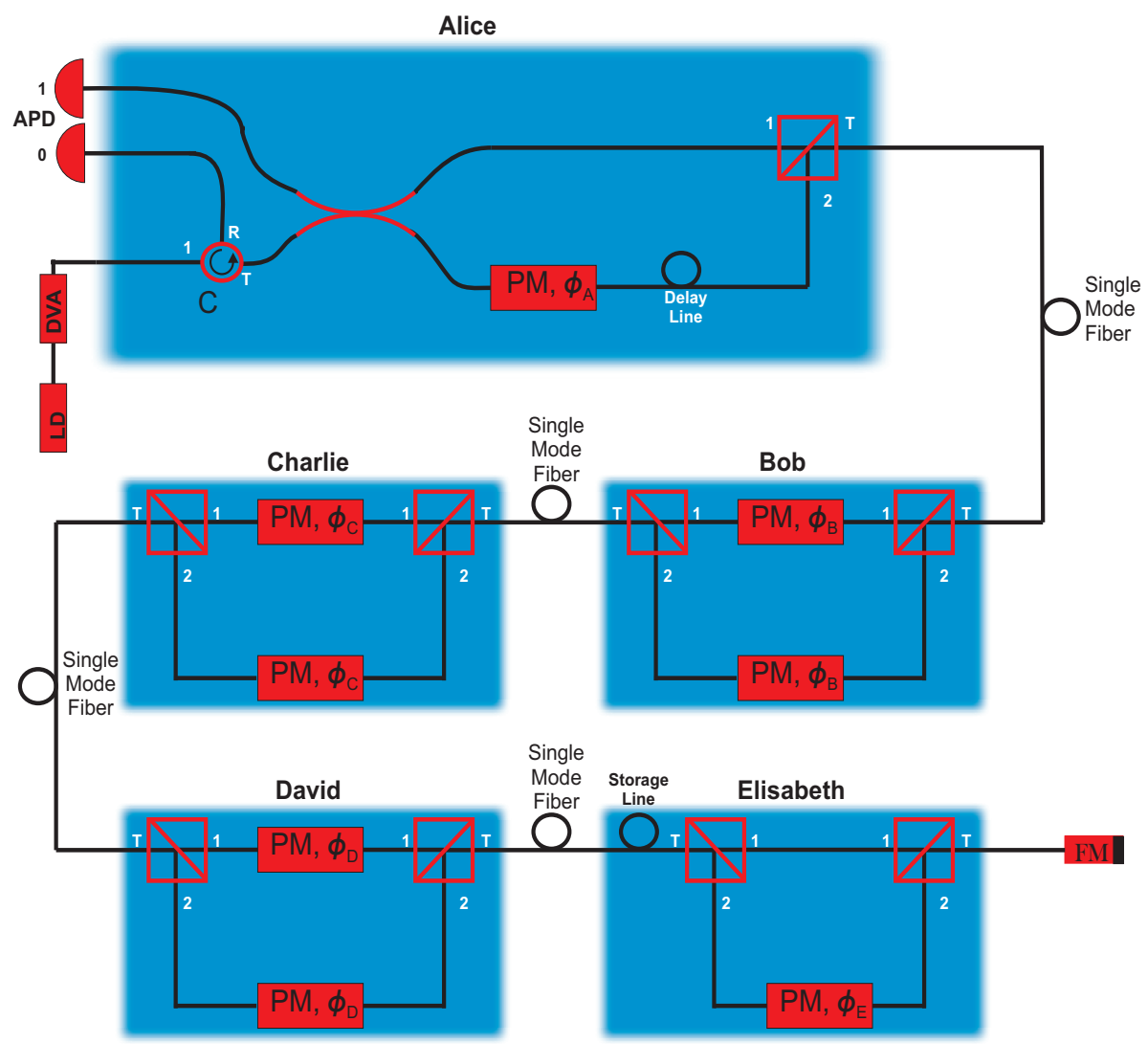

Figure 6.11: Scheme of the Quantum Secret Sharing Plug and Play setup. 


\section{Chapter 7}

\section{Experimental Components}

Here we present the most important and used components in the experimental part of the thesis.

\subsection{Pulse Generator}

The pulse generator used in the experiment is a Quantum Composer, with a maximum frequency of $20 \mathrm{MHz}$, with both TTL output (for Laser and APD's) and Variable 0-12 Voltage output (for phase modulators). This pulse generator lets one use different signal output modes. The optional modes are Continuous Mode, Burst Mode (with Wait) and Duty cycle. The most commonly used setup in the experiment with the presence of a storage line can be viewed in table 7.1 and fig. 7.2,

\begin{tabular}{l|l}
\hline \hline Master Clock & Duty Cycle \\
\hline Laser, Phase Modulators, APD's & Burst and Wait \\
\hline
\end{tabular}

Table 7.1: Most common settings in experiment.

The burst mode is set to the number of pulses that can fit inside the storage line. The "Wait" option, lets a channel to wait a certain number of pulses (the time in units of seconds depend on which frequency one have set the Master Clock to run, e.g. 1 pulse wait @ $1 \mathrm{MHz}=1 \mu \mathrm{s}$ or 1 pulse wait @ $2 \mathrm{MHz}=0.5 \mu \mathrm{s}$ etc.). As long as one waits less than the duty cycle for the Master Clock, one will not have any "rate" problems, which one will have if e.g. let pulse width exceed $\mathrm{T}=1$ /frequency. The disadvantages of this pulse generator is its long rise time for Variable Voltage outputs, which is $\approx 100 \mathrm{~ns}$ (see fig. 7.1 ), and its resolution of only $250 \mathrm{ps,} \mathrm{which} \mathrm{means} \mathrm{that}$ delays cannot be set with less step-size than $250 \mathrm{ps}$. However, this is not a problem, since the APD's provide a resolution of $10 \mathrm{ps}$. The long rise time 
of the pulse generator limits the laser's pumping frequency, since parties will then need some time to raise their pulses for phase modulation. An example of some pulse settings can be seen in fig. 7.2.

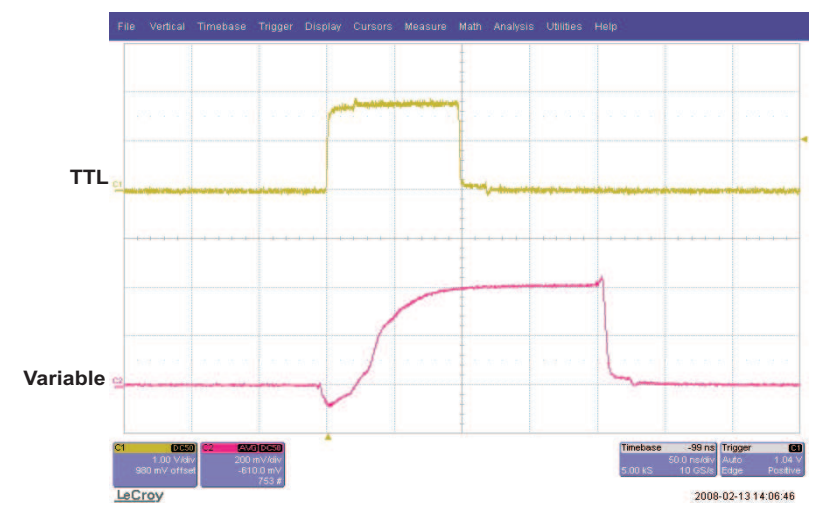

Figure 7.1: The rise time of the TTL signal is much shorter than the rise time for the variable output.

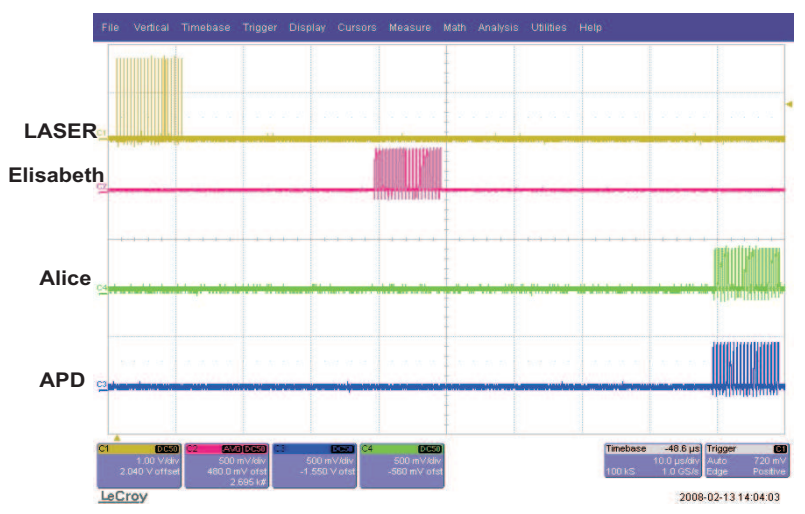

Figure 7.2: An illustration of the pulse generator's different outputs. Timings and voltages should not be regarded as literary. 


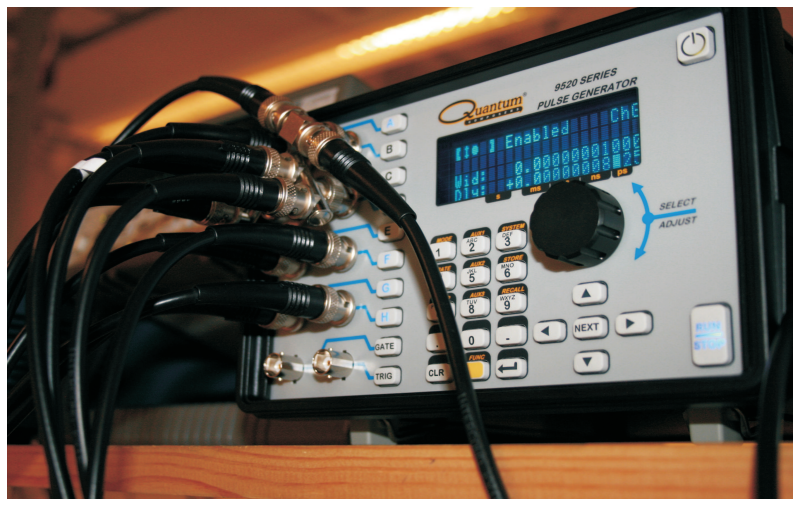

Figure 7.3: Quantum Composer Pulse Generator.

\subsection{Laser Source}

The laser sources used in the experiments were $1550 \mathrm{~nm}$ pumped laser diodes. In fig. 7.4 we can see that the laser output is attached to an isolator and a horizontal polarizer. The isolator is present because of backscattered light from the attenuator attached after the laser. The polarizer lets only horizontal polarized light to transmit.

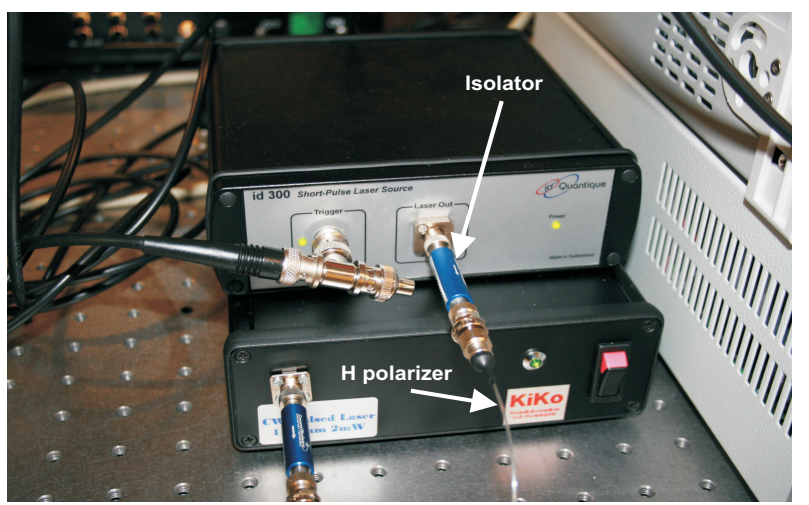

Figure 7.4: $1550 \mathrm{~nm}$ pulsed laser with isolator and polarizer.

\subsection{Digital Variable Attenuator}

The digital variable attenuator which we used in the experiment ranges from 0 to $62 \mathrm{~dB}$ attenuation, with an in-linearity starting at around $12 \mathrm{~dB}$. I.e. one have to add $\approx 0.3 \mathrm{~dB}$ when wanting to attenuate in the range of 12 $25 \mathrm{~dB}, \approx 0.5 \mathrm{~dB}$ in the range of $25-35 \mathrm{~dB}$ and $\approx 0.6 \mathrm{~dB}$ in the range of 35 $-45 \mathrm{~dB}$. 


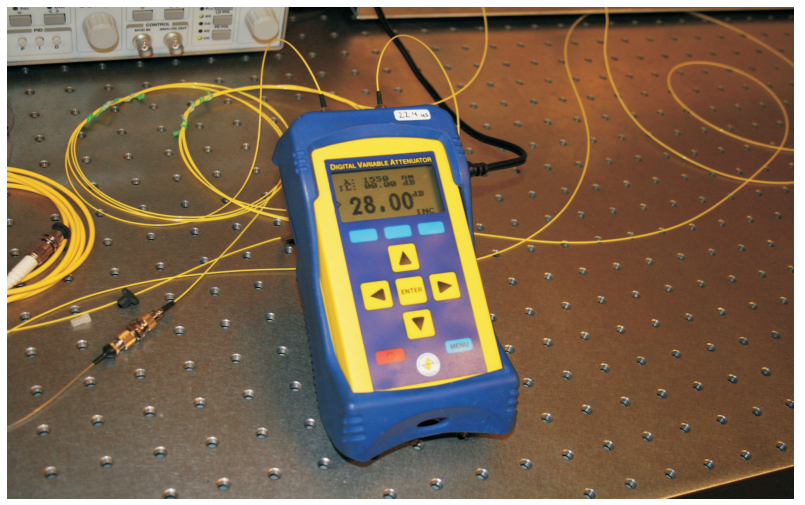

Figure 7.5: Optical attenuator.

\subsection{Parties}

After we had assembled each station properly we built them inside boxes, making the components less fragile and providing higher mobility.

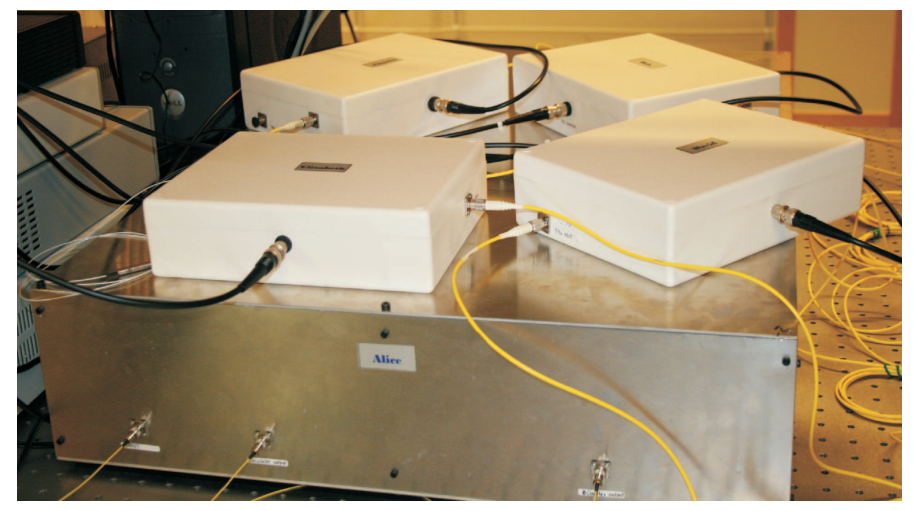

Figure 7.6: All 5 parties, with Alice (the interferometer) in the bottom.

\subsection{Fiber spools}

In order to simulate real distances between parties, we used single mode fiber spools of various lengths. 


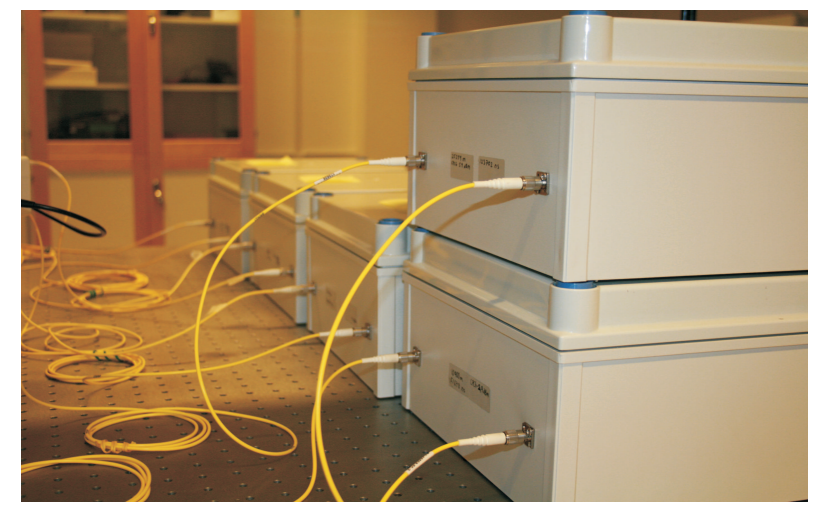

Figure 7.7: Fiber spools between parties.

\subsection{Optical switches}

We also built in the pig-tailed optical switches into boxes.

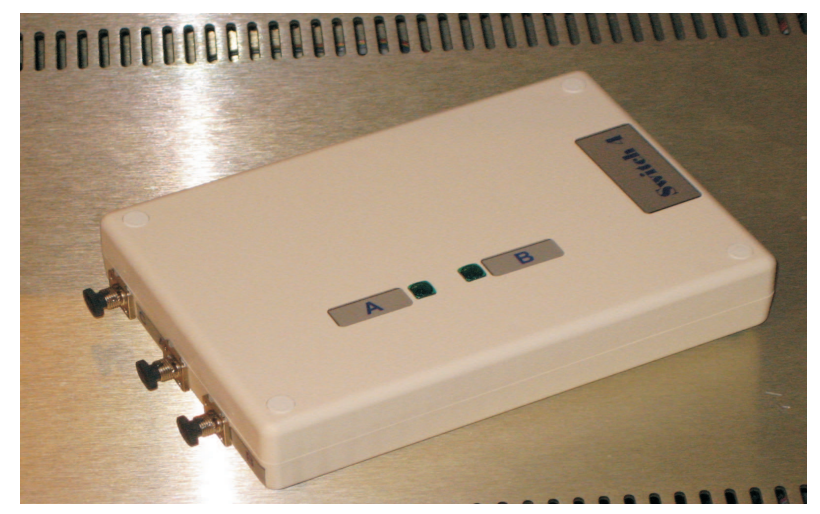

Figure 7.8: Optical switch.

\subsection{Low-noise photo-diode}

We assembled a photo-diode and an signal amplifier in series, in order to be able to detect pulses that have a very low signal power. 


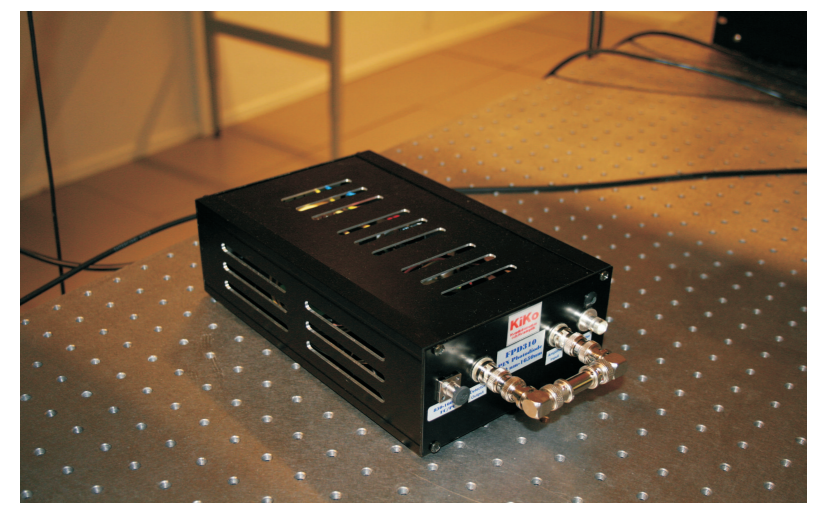

Figure 7.9: Low-noise photo-diode.

\subsection{Single Photon Detectors}

The single photon detectors used in the experiments are Princeton Lightwave InGaAs APD's (avalanche photo diodes). The APD's provide (in gated mode) a dark count rate of $\approx(1-3) \times 10^{-5} /$ bias pulse (depending on individual detector). "Per bias pulse" simply means per gate opened for photon detection, so higher frequencies give more dark counts per second. The gate window is $1 \mathrm{~ns}$. However, the APD's provide a delay option in steps of 10 ps which is very useful since our pulse generator provide a resolution of only 250 ps.

Attempts have been made to decrease the bias voltage in order to lower the dark count probability. Although we were fully aware of the fact that this would also decrease the quantum efficiency of the detector, we were hoping that the relation between these two decreasing factors would not be fully linear and that there would exist a trade-off in favor of the quantum efficiency. However, the measurements examining this relationship shows a linear relation, see fig. 7.10. 

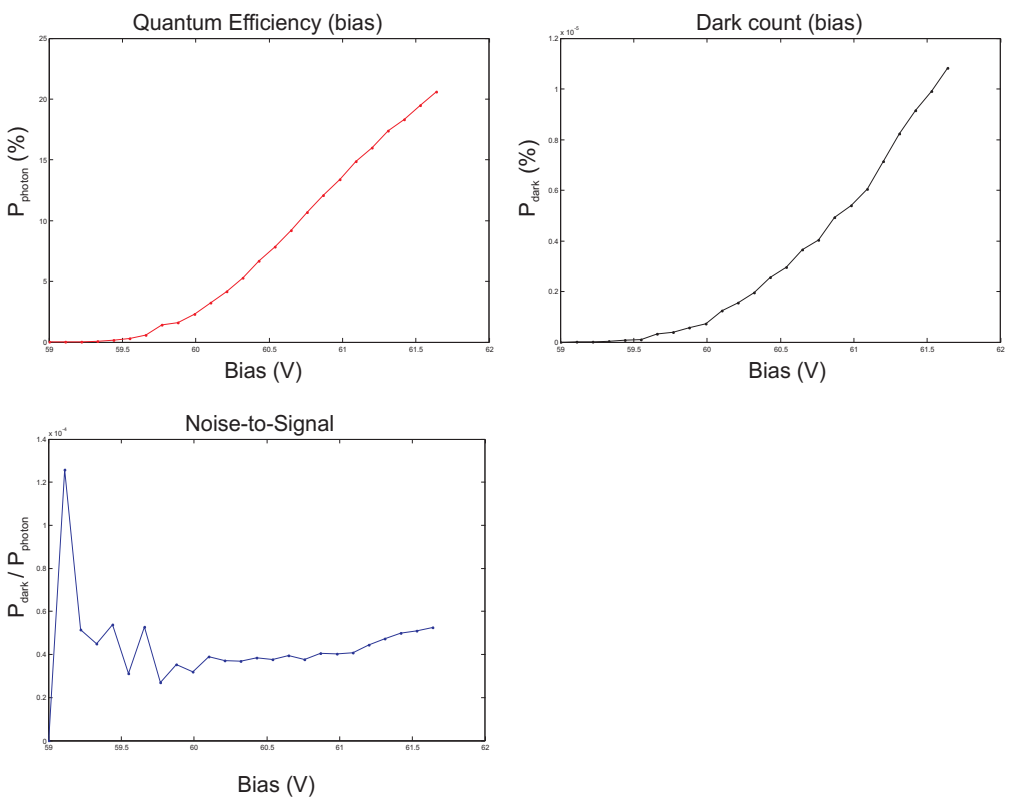

Figure 7.10: Plots over detection probability of photon and dark count as function of bias voltage of the detector. The large fluctuations at lower bias voltages in the ratio plot are simply due to low time-frame measurement relative probabilities.

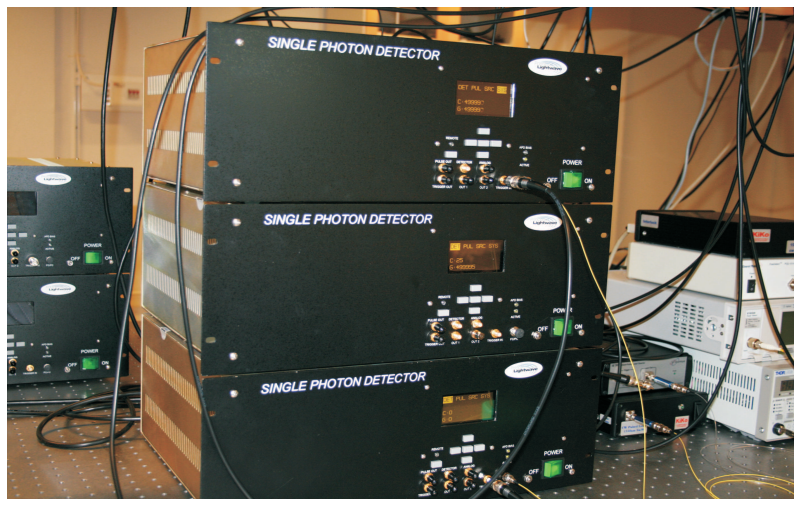

Figure 7.11: Single photon detectors. 


\section{Chapter 8}

\section{Experimental Results}

\subsection{Multi-User QKD}

The optical switches used in the experiments are manually controlled switches, with an insertion loss of $\approx 1 \mathrm{~dB}$ attenuation from one port to the other. In the star configuration, all optical switches are controlled by Alice. However in the tree configuration, two of these are controlled by Charlie who is obeying Alice's command.

The raw rate measured in the experiments are given in tables 8.1 and 8.2. These are measured by keeping both phase modulators off, measuring only constructive interference on bit 1 (APD 1) and subtracting dark counts, also they are already halved as $q=1 / 2$ in eq. 5.4. Visibilities are measured (using eq. 5.7) as the difference between maximum counts on one detector when both phase modulators are off and when adding a phase shift of $\pi$ through one of the stations. It is in general the end-users' phase modulators which are adding this phase shift, since the end-users' stations are more demanding regarding the timing of the modulation pulse. Also, end-users are sensitive regarding this phase shift, (each phase modulator have a specific voltage requirement for a $\pi$ phase shift, called $V_{\pi}$ ). We have tried to match all phase modulators in the best way possible (recall the first parameter which said that phase modulators need to match in attenuation). Even if both modulators in a station have (according to their data sheet) identical $V_{\pi}$, one still want to regard the end-user setup as the weakest link for visibility measurement. This argument does not exclude Elisabeth. Since both parts of the short arm pulse are not phase modulated at the same point in time, it is not given that the $V_{\pi}$ is completely unchanged during the time difference, due to electronics. Also, uncertainties calculated are statistical errors. 


\subsubsection{Tree Configuration}

The tree-like network is rather efficient economically. Since some parties may be geographically grouped, there is no need for Alice to set up an independent quantum channel to each member. Instead, some parts of this channel can be used for the grouped users. Results for the tree configuration can be seen below, in table 8.1 .

\begin{tabular}{ccccc}
\hline \hline & $\mathrm{L}(\mathrm{km})$ & QBER det & Visibility (\%) & $R_{\text {raw }}(\mathrm{bit} / \mathrm{s})$ \\
\hline Bob & 50 & $4.37 \pm 0.03$ & $99.07 \pm 0.01$ & 62.7 \\
Charlie & 25.3 & $1.24 \pm 0.01$ & $99.17 \pm 0.01$ & 295 \\
David & 44.3 & $4.95 \pm 0.03$ & $98.99 \pm 0.01$ & 58.9 \\
Elisabeth & 44.3 & $5.04 \pm 0.03$ & $99.12 \pm 0.02$ & 57.8 \\
\hline
\end{tabular}

Table 8.1: Table of measurement results for the tree configuration with mean photon number $\mu=0.1$. Distances denote the total fiber length between Alice and user. Also, the total error rate is not being shown, instead the two main contributing factors are displayed (Visibility and $Q B E R_{d e t}$ ).

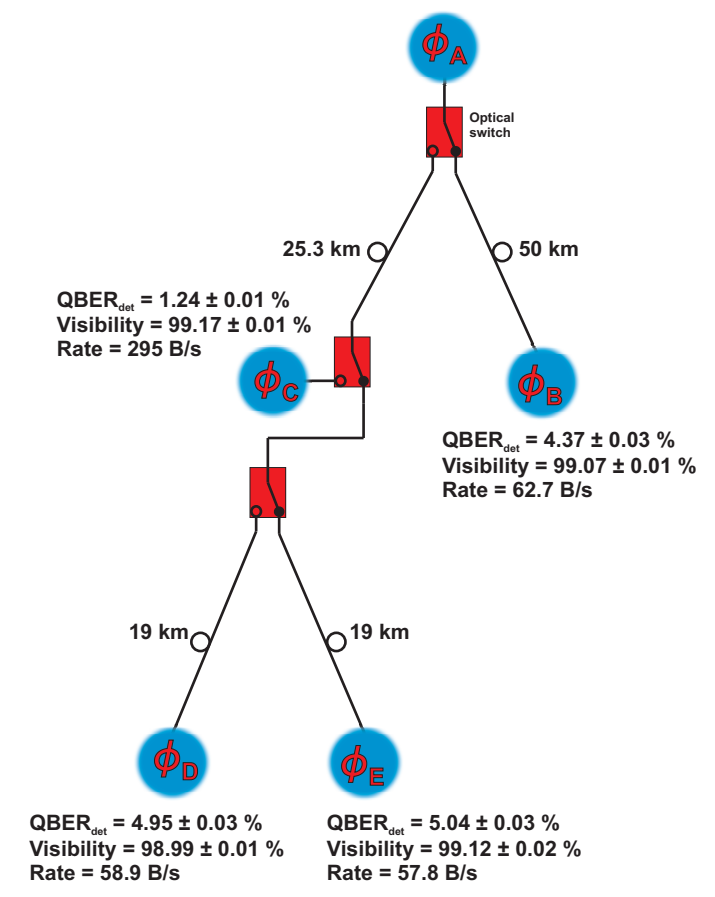

Figure 8.1: Measurement results for the tree configuration

It might seem confusing when David and Elisabeth show higher error rate $\left(Q B E R_{\text {det }}\right)$ than Bob, even though the distances from Alice to David and Elisabeth are shorter than the distance from Alice to Bob. The reason 
for this effect is because of two contributing factors. One of them being the two extra optical switches that lay in the path of David and Elisabeth providing additional attenuation. The other reason is the number of fiber spools connected. Bob's fiber link consists of one long $50 \mathrm{~km}$ fiber spool, whereas David and Elisabeth's fiber link consist of two fiber spools, one to Charlie and one from Charlie to Alice. Throughout the experimental part of the thesis, the connection of several fiber spools, which all together provide a higher attenuation than a single spool (in terms of attenuation per kilometer), has been an underlying effect one has to take in to account when comparing with theoretical calculations. This is of course due to loss in the connectors.

\subsubsection{Star Configuration}

Here we setup a star-like network, where all optical switches are placed within an arms distance of Alice. Protocol and applicability is completely analogous to the tree-like network.

\begin{tabular}{ccccc}
\hline \hline & $\mathrm{L}(\mathrm{km})$ & $Q B E R_{\text {det }}$ & Visibility $(\%)$ & $R_{\text {raw }}(\mathrm{bit} / \mathrm{s})$ \\
\hline Bob & 45.2 & $4.62 \pm 0.03$ & $98.86 \pm 0.03$ & 59.3 \\
Charlie & 50 & $5.56 \pm 0.03$ & $99.14 \pm 0.01$ & 51.0 \\
David & 44.3 & $3.57 \pm 0.02$ & $98.76 \pm 0.02$ & 81.7 \\
Elisabeth & 44.3 & $4.69 \pm 0.03$ & $99.11 \pm 0.02$ & 62.2 \\
& 59.4 & $10.26 \pm 0.08$ & $99.10 \pm 0.01$ & 24.5 \\
\hline
\end{tabular}

Table 8.2: Table of measurement results for the star configuration with mean photon number $\mu=0.1$. Distances denote the total fiber length between Alice and user. Also, the total error rate is not being shown, instead the two main contributing factors are displayed (Visibility and $Q B E R_{d e t}$ ). 


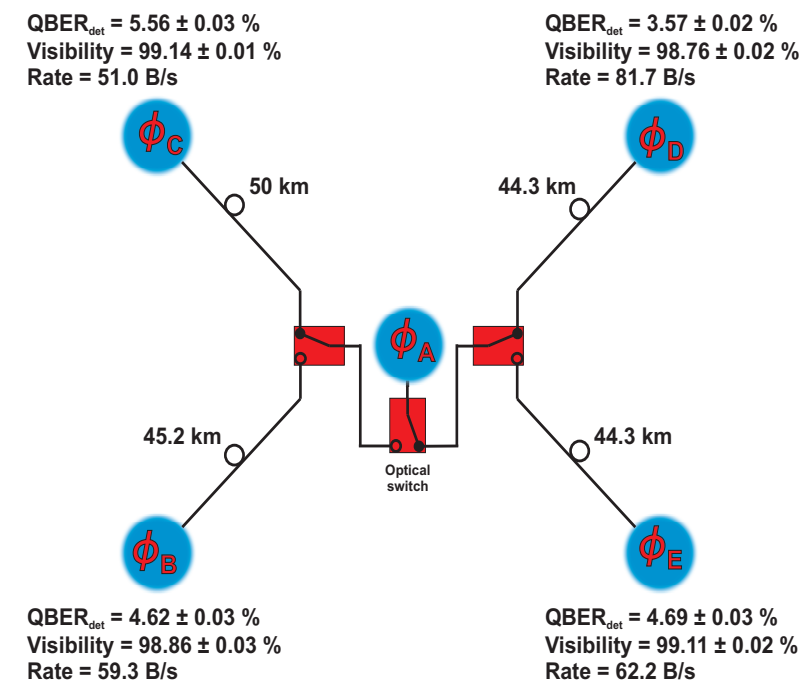

Figure 8.2: Measurement results for the star configuration

Table. 8.2 might also be somewhat confusing. For instance, one of Elisabeth's measurements compares to David's (in the sense of distance). Inspite of this fact, their error rate differ. This is because they use different fiber spools with different attenuation per kilometer. However, both of these result should be compared to the theoretical one, which in fact assume 0.25 $\mathrm{dB} / \mathrm{km}$. The results are discussed further after we have presented the experimental data for Quantum Secret Sharing.

\subsection{Quantum Secret Sharing}

The Quantum Secret Sharing experiment was carried out for 3, 4 and 5 parties. Method of measurements are practically the same as described in Multi-user QKD. For 3 and 4 party implementation, we were able to make measurements for different distances, following a theoretical curve given by eq. 5.9 for the error rate $Q B E R_{\text {det }}$ and eq. 5.4 for the raw rate $R_{\text {raw }}$. The parties excluded in the 3 and 4 party experiment were carefully selected through their respective attenuation. The experiment on 5 party implementation was carried out for one single total distance of $6.7 \mathrm{~km}$. Also, uncertainties calculated are statistical errors.

\subsubsection{Parties}

The 3-party experiment is very similar to the 4-party experiment, except for the absence of Charlie. Here, we carried out the measurements for different total distances of $40.8 \mathrm{~km}, 46.3 \mathrm{~km}, 50.5 \mathrm{~km}, 55.5 \mathrm{~km}, 61.0 \mathrm{~km}$ and 66.1 
$\mathrm{km}$. The mutual distances between parties and the total distances are given in table 8.3. The error rates and visibilities are given in table 8.4 and the rates are given in table 8.5 .

\begin{tabular}{lcc}
\hline \hline $\mathrm{A} \rightarrow \mathrm{D}(\mathrm{km})$ & $\mathrm{D} \rightarrow \mathrm{E}(\mathrm{km})$ & $\mathrm{L}(\mathrm{km})$ \\
\hline $10.559+5.0$ & 25.259 & 40.8 \\
$10.559+5.0+5.549$ & 25.259 & 46.3 \\
25.259 & 25.259 & 50.5 \\
$25.259+5.0$ & 25.259 & 55.5 \\
$25.259+5.0+5.549$ & 25.259 & 61.0 \\
$25.259+10.559+5.0$ & 25.259 & 66.1 \\
\hline
\end{tabular}

Table 8.3: Table over distances between parties from the 3 party secret sharing experiment. $\mathrm{L}$ denotes the total fiber length in setup.

\begin{tabular}{ccccc}
\hline \hline $\mathrm{L}(\mathrm{km})$ & $\begin{array}{c}Q B E R_{\text {det }} \\
(\mu=0.1)\end{array}$ & $\begin{array}{c}\text { QBER det } \\
(\mu=0.2)\end{array}$ & $\begin{array}{c}\text { QBER } \\
(\mu=0.3)\end{array}$ & Visibility $(\%)$ \\
\hline 40.8 & $5.29 \pm 0.02$ & $2.68 \pm 0.01$ & $1.759 \pm 0.004$ & $99.05 \pm 0.01$ \\
46.3 & $8.17 \pm 0.04$ & $4.21 \pm 0.01$ & $2.714 \pm 0.006$ & $99.16 \pm 0.01$ \\
50.5 & $7.13 \pm 0.04$ & $3.58 \pm 0.01$ & $2.44 \pm 0.03$ & $99.02 \pm 0.01$ \\
55.5 & $12.43 \pm 0.06$ & $6.11 \pm 0.03$ & $3.82 \pm 0.02$ & $98.51 \pm 0.03$ \\
61.0 & $13.05 \pm 0.11$ & $6.59 \pm 0.03$ & $4.32 \pm 0.02$ & $98.87 \pm 0.04$ \\
66.1 & $21.99 \pm 0.27$ & $10.71 \pm 0.08$ & $7.07 \pm 0.05$ & $99.03 \pm 0.04$ \\
\hline
\end{tabular}

Table 8.4: Measurement data of $Q B E R_{\text {det }}$ and visibility from the 3 party secret sharing experiment. 


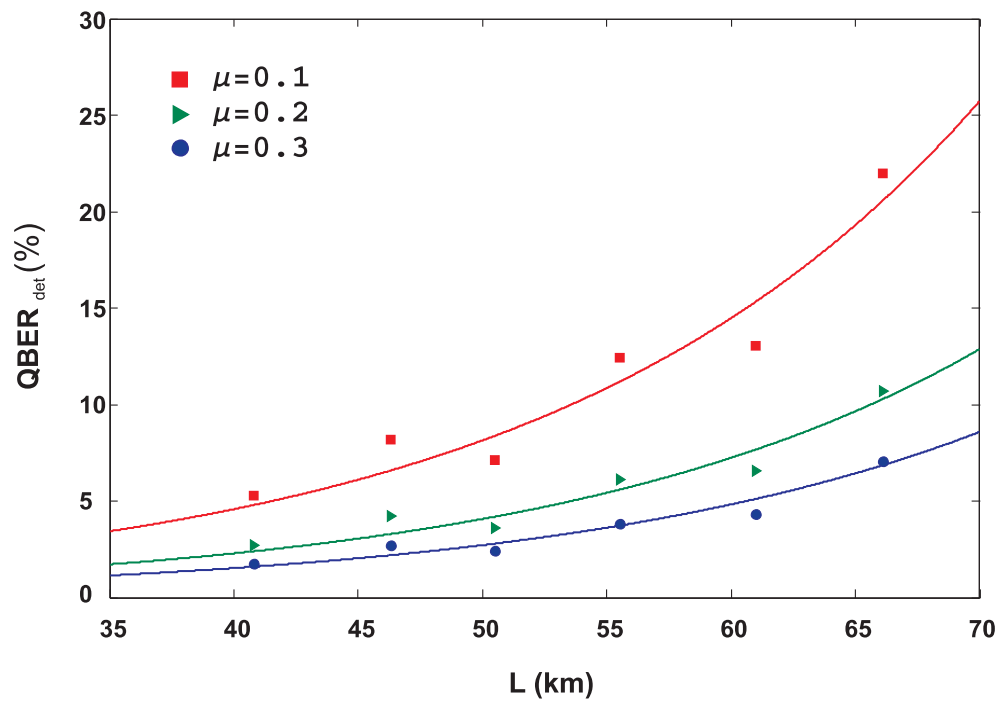

Figure 8.3: Plot over experimental data of $Q B E R_{\text {det }}$ as a function of the total distance $\mathrm{L}$, from the 3 party secret sharing experiment. The solid lines correspond to theoretical values while the dots (square, triangle and circle) are the experimental values.

\begin{tabular}{cccc}
\hline \hline $\mathrm{L}(\mathrm{km})$ & $\begin{array}{c}R_{\text {raw }}(\mathrm{B} / \mathrm{s}) \\
(\mu=0.1)\end{array}$ & $\begin{array}{c}R_{\text {raw }}(\mathrm{B} / \mathrm{s}) \\
(\mu=0.2)\end{array}$ & $\begin{array}{c}R_{\text {raw }}(\mathrm{B} / \mathrm{s}) \\
(\mu=0.3)\end{array}$ \\
\hline 40.8 & 73.4 & 144.7 & 220.7 \\
46.3 & 45.8 & 88.9 & 137.7 \\
50.5 & 51.1 & 101.8 & 149.3 \\
55.5 & 28.3 & 57.6 & 91.9 \\
61.0 & 26.1 & 51.6 & 78.7 \\
66.1 & 15.0 & 30.7 & 46.6 \\
\hline
\end{tabular}

Table 8.5: Measurement data of raw rates $R_{\text {raw }}$ from the 3 party secret sharing experiment. 


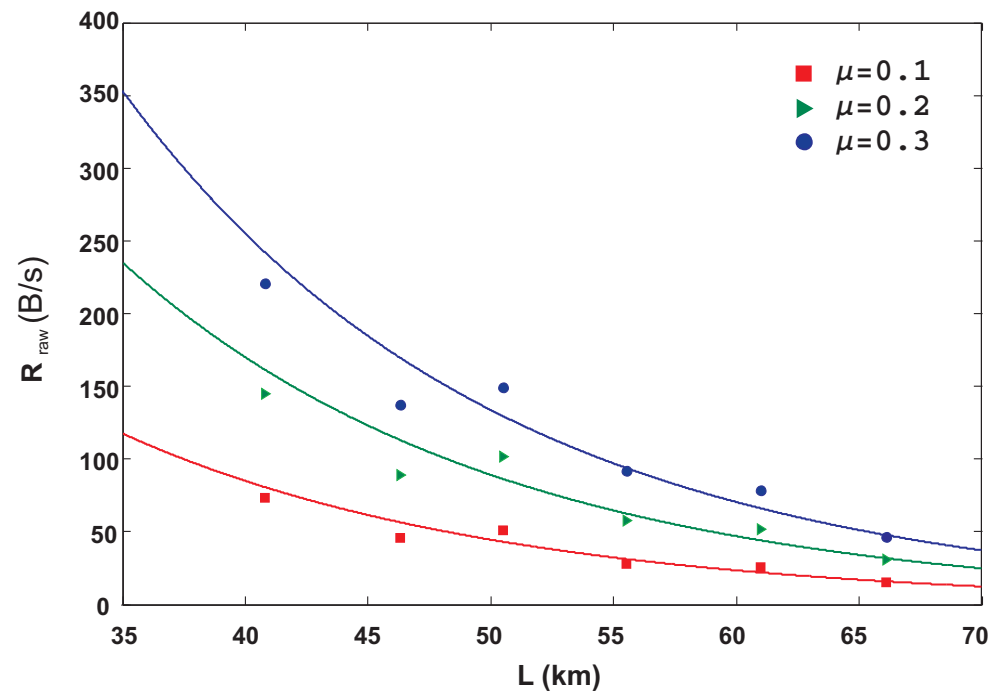

Figure 8.4: Plot over experimental data of $R_{\text {raw }}$ as a function of the total distance L, from the 3 party secret sharing experiment. The solid lines correspond to theoretical values while the dots (square, triangle and circle) are the experimental values.

\subsubsection{4 parties}

For the 4-party experiment, we were also able to follow a theoretical curve. This time, only without the presence of Bob, since his station has the highest insertion loss. We carried out the measurement for 6 different total distances, $16.6 \mathrm{~km}, 21 \mathrm{~km}, 26.5 \mathrm{~km}, 30.6 \mathrm{~km}, 36.5 \mathrm{~km}$ and $40.5 \mathrm{~km}$. The mutual distances between parties and the total distances are given in table 8.6. The error rates and visibilities are given in table 8.7 and the rates are given in table 8.8.

\begin{tabular}{cccc}
\hline \hline $\mathrm{A} \rightarrow \mathrm{C}(\mathrm{km})$ & $\mathrm{C} \rightarrow \mathrm{D}(\mathrm{km})$ & $\mathrm{D} \rightarrow \mathrm{E}(\mathrm{km})$ & $\mathrm{L}(\mathrm{km})$ \\
\hline 10.559 & 5.0 & 1.0 & 16.6 \\
10.559 & 5.0 & 5.459 & 21.0 \\
10.559 & 5.0 & 10.9 & 26.5 \\
10.559 & 5.0 & 15.059 & 30.6 \\
10.559 & 10.9 & 15.059 & 36.5 \\
10.559 & 10.9 & 19.057 & 40.5 \\
\hline
\end{tabular}

Table 8.6: Table over distances between parties from the 4 party secret sharing experiment. L denotes the total fiber length in setup. 


\begin{tabular}{ccccc}
\hline \hline L $(\mathrm{km})$ & $\begin{array}{c}Q B E R_{\text {det }} \\
(\mu=0.1)\end{array}$ & $\begin{array}{c}Q B E R_{\text {det }} \\
(\mu=0.2)\end{array}$ & $\begin{array}{c}\text { QBER } \\
(\mu=0.3)\end{array}$ & Visibility (\%) \\
\hline 16.6 & $7.28 \pm 0.03$ & $3.66 \pm 0.01$ & $2.50 \pm 0.01$ & $98.93 \pm 0.01$ \\
21.0 & $7.76 \pm 0.05$ & $3.85 \pm 0.01$ & $2.57 \pm 0.01$ & $98.93 \pm 0.02$ \\
26.5 & $8.64 \pm 0.07$ & $4.52 \pm 0.02$ & $3.61 \pm 0.02$ & $98.92 \pm 0.03$ \\
30.6 & $15.67 \pm 0.08$ & $7.74 \pm 0.04$ & $5.15 \pm 0.02$ & $99.12 \pm 0.04$ \\
36.5 & $17.73 \pm 0.22$ & $9.12 \pm 0.07$ & $6.23 \pm 0.04$ & $98.97 \pm 0.03$ \\
40.5 & $23.11 \pm 0.23$ & $10.16 \pm 0.07$ & $6.62 \pm 0.05$ & $98.79 \pm 0.06$ \\
\hline
\end{tabular}

Table 8.7: Measurement data of $Q B E R_{\text {det }}$ and visibility from the 4 party secret sharing experiment.

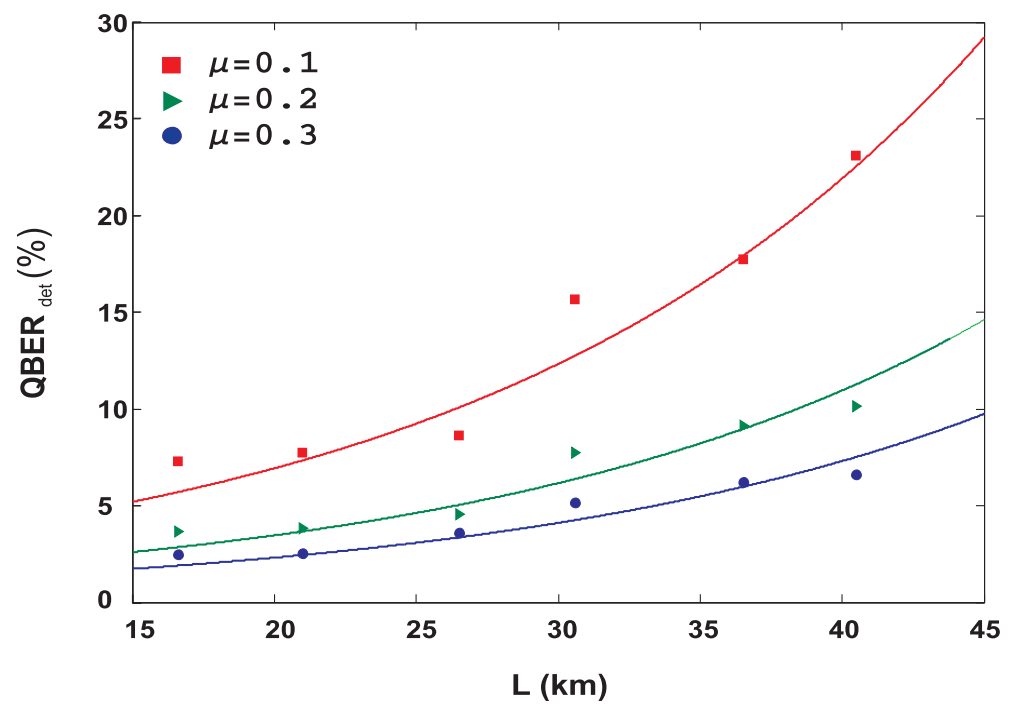

Figure 8.5: Plot over experimental data of $Q B E R_{\text {det }}$ as a function of the total distance $\mathrm{L}$, from the 4 party secret sharing experiment. The solid lines correspond to theoretical values while the dots (square, triangle and circle) are the experimental values.

\begin{tabular}{cccc}
\hline \hline $\mathrm{L}(\mathrm{km})$ & $\begin{array}{c}R_{\text {raw }}(\mathrm{B} / \mathrm{s}) \\
(\mu=0.1)\end{array}$ & $\begin{array}{c}R_{\text {raw }}(\mathrm{B} / \mathrm{s}) \\
(\mu=0.2)\end{array}$ & $\begin{array}{c}R_{\text {raw }}(\mathrm{B} / \mathrm{s}) \\
(\mu=0.3)\end{array}$ \\
\hline 16.6 & 64.4 & 127.9 & 187.0 \\
21.0 & 58.0 & 116.7 & 175.2 \\
26.5 & 50.0 & 95.4 & 119.7 \\
30.6 & 26.7 & 54.0 & 81.2 \\
36.5 & 22.7 & 44.1 & 64.5 \\
40.5 & 16.9 & 38.5 & 59.1 \\
\hline
\end{tabular}

Table 8.8: Measurement data of raw rates $R_{\text {raw }}$ from the 4 party secret sharing experiment. 


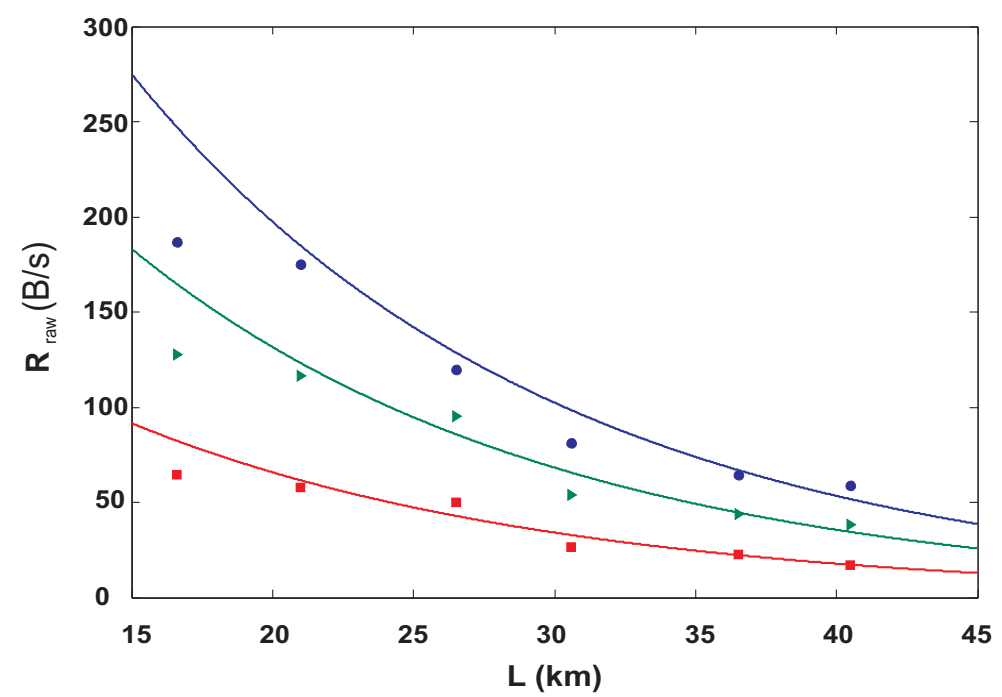

Figure 8.6: Plot over experimental data of $R_{\text {raw }}$ as a function of the total distance $\mathrm{L}$, from the 4 party secret sharing experiment. The solid lines correspond to theoretical values while the dots (square, triangle and circle) are the experimental values.

\subsubsection{Parties}

The experiment on 5 party implementation was carried out for one single total distance of $6.7 \mathrm{~km}$ only, since already at this distance we were close to the maximum allowed $Q B E R$ of $11 \%$ for unconditionally secure quantum key sharing ${ }^{1}$. The two main reasons for this is the attenuation provided by party stations which range between $4.2-5 \mathrm{~dB}$. This attenuation correspond to $\approx 20 \mathrm{~km}$. Also, when using short fiber links $(200 \mathrm{~m}, 500 \mathrm{~m}, 1000 \mathrm{~m}$ and $5000 \mathrm{~m}$ ), the loss in the connectors become the main factor of attenuation rather than the loss in the fiber itself. For instance, each of these short fiber spools (including interconnecting fibers to stations) provide an attenuation $\leq 1.6 \mathrm{~dB}$ corresponding to a total of $12.4 \mathrm{~km}(3.1 \mathrm{~dB})$, whereas the fiber itself should provide not more than $0.25 \mathrm{~dB}$ (for the $1 \mathrm{~km}$ spool). A theoretical value of the $Q B E R_{\text {det }}$ is easily calculated, given the attenuation of party stations, Alice $=5 \mathrm{~dB}$, Bob $=5 \mathrm{~dB}$, Charlie $=4.5$ and David $=4.2 \mathrm{~dB}$, and assuming $0.25 \mathrm{~dB} / \mathrm{km}$, see eq. 8.1. Experimental results can be viewed below in table 8.9 .

$$
\left\{\begin{array}{l}
Q B E R_{\text {det }}=\frac{p_{\text {noise }}}{p_{\text {photon }}}=\frac{1.1 * 10^{-5}}{\mu \epsilon \eta_{\text {link }}} \\
\eta_{\text {link }}=10^{-(0.25 * 6.7+5+5+4.5+4.2) / 10 \Rightarrow} \Rightarrow Q B E R_{\text {det }}=6.2 \% \\
\mu=0.1 \\
\epsilon=0.2
\end{array}\right.
$$

\footnotetext{
${ }^{1}$ Although, this limit has not yet been proven for quantum secret sharing.
} 


\begin{tabular}{l|lcc|l}
\hline \hline $\mathrm{L}(\mathrm{km})$ & & $Q B E R_{\text {det }}$ & $R_{\text {raw }}(\mathrm{B} / \mathrm{s})$ & Visibility (\%) \\
\hline $6.7 \mathrm{~km}$ & $\mu=0.1$ & $8.72 \pm 0.05$ & 55.1 & $98.95 \pm 0.03$ \\
& $\mu=0.2$ & $4.54 \pm 0.01$ & 105.9 & \\
& $\mu=0.3$ & $2.75 \pm 0.01$ & 174.8 & \\
\hline
\end{tabular}

Table 8.9: Measurement data from the 5 party secret sharing experiment.

We have indeed a difference between the theoretical value and the experimental one. However, the difference is well acceptable for above mentioned reasons. Also, it should be emphasized that a similar measurement was carried out with another detector with higher dark count probability and resulted in $20 \%$ error rate for $\mu=0.1$.

\subsection{Discussion}

This discussion apply to both the measurement for Multi-user QKD and for Quantum Secret Sharing.

Since we have carefully measured the attenuation of every component, we can predict the expected theoretical values in a convincing way. As mentioned before, we have assumed $0.25 \mathrm{~dB} / \mathrm{km}$ (attenuation) through our fiber spools. However, one need also to take into account the losses in fiber connectors connecting a station to a fiber spool, or connecting a fiber spool to another fiber spool. We have estimated $\approx 0.3 \mathrm{~dB}$ attenuation per connector, adding this to eq. 8.1. Since we carry out no stabilizing procedure regarding the interferometer or any other component, the high visibilities achieved are realized due to the Plug and Play implementation and carefully measured and selected components in the setup. Except the values of total distances, $Q B E R_{\text {det }}$ and visibilities we have also presented the raw rate, $R_{\text {raw }}$. These raw rates however are not values to be regarded as limited by the setup, since they are only limited by our pulse generator which have a long rise time for the phase modulating pulses. Since we are using a single pulse generator to handle all units in the setup, the rise time sets a practical limit of $\approx 2 \mathrm{MHz}$. The lower frequency, the less one have to struggle with the timings for all units and above $2 \mathrm{MHz}$ it is not obvious that it is even possible to time all units correctly. Of course on could put some units to passive mode, disabling them from taking any participation of the protocol, but somehow this does not seem convincing. 


\section{Part III}

Conclusion 
We have studied the feasibility of a 3, 4 and 5 party Single Qubit Quantum Secret Sharing with phase encoding implementation over birefringent single mode fiber. We have reached total distances of $66.1,40.5$ and $6.7 \mathrm{~km}$ for 3,4 and 5 parties respectively with error rates below the limit for unconditional secure quantum communication protocol. Although the accepted upper limit of Eve's gained knowledge about the information in QKD, for which it was $11 \%$ has been proven, Quantum Secret Sharing yet awaits such a proof. During the experimental part of this thesis, we have assumed the same level of security for Quantum Secret Sharing. Visibilities were $\geq 98.5 \%$ and the error rates $Q B E R_{\text {det }}$ were given with $\mu=0.1,0.2$ and 0.3 . We have also studied the feasibility of a Multi User Quantum Key Distribution Network with 5 nodes (users), over optical switches and birefringent single mode fibers in both star and tree configuration. Here, we have reached a maximum distance of $59.4 \mathrm{~km}$ with an error rate below the limit of $11 \%$ with $\mu=0.1$, and the visibilities were $\geq 98.8 \%$. Phase encoding implementation with weak-coherent states (faint pulses), has been shown to be very practical for the realization of the two above mentioned implementations, along with the Plug and Play setup. To our best knowledge, this is the first time an experimental 5 party Single Qubit Quantum Secret Sharing with phase encoding protocol has been realized. Also, our 5 node Multi User QKD Network experiments are well competitive, considering published articles about similar networks [21][22].

We have mostly discussed the stability of our setup regarding interferometric fidelity. There is another stability which is important when using weak coherent states. Consider the Quantum Secret Sharing setup. Since single mode fiber is birefringent and standard phase modulators are polarization sensitive, aligned to the horizontal polarization, the attenuation the pulse experience on its way to Elisabeth will fluctuate, disabling Elisabeth (or Alice) to attenuate the pulse to a certain $\mu$. Also, Alice is not able to apriori determine any expected counts on her APD's. However, when using polarization insensitive stations as presented in this report, the stability of the attenuation related to polarization is guaranteed.

Considering the setups and components used in the experiment, regarding attenuation, stability and efficiency, we are convinced that we have achieved the greatest data possible with the available hardware. However, applying our current setup in a field experiment will need further improvements, such as automatic synchronization of phase modulators. This can be done by sending a strong laser pulse on another wavelength which a user can use to synchronize her phase modulator with, so called WavelengthDivision-Multiplexing (WDM). These improvements may seem fancy for a lab experiment, but are necessary in field experiments. 
We have not discussed the security analysis of quantum communication protocols in any depth, since this requires a significant amount of analysis, corresponding at least to an entire Master thesis. However, we have presented the best known and most famous types of attacks such as Photon Number Splitting (PNS) and Intercept and Resend (I-R) attacks. 


\section{Part IV}

\section{Appendix A}


The attenuation $\mathrm{A}$, given in decibel with unit $\mathrm{dBm}$, is related to the power $\mathrm{P}$, given in unit of watt, through $A=10 \log _{10}\left(\frac{P}{1 \mathrm{~mW}}\right)$ and since our power meter returns the value $\mathrm{A}$ in units of $\mathrm{dBm} / \mathrm{s}$, it is important to know the frequency, since we are using pulsed laser and want information about the power in a single pulse. The attenuation A, for single photon level, with average number of photons $\mu$, is given by,

$$
\begin{gathered}
A_{\text {photon }}=10 \log _{10} \frac{P}{1 m W}=10 \log _{10} \frac{\mu E f}{1 m W}=10 \log _{10} \frac{\mu h c f}{\lambda \times 1 m W}= \\
=-112 d B m, \mu=0.1, f=500 k H z, \lambda=1550 \mathrm{~nm} \\
=-109 d B m, \mu=0.1, f=1 M H z, \lambda=1550 \mathrm{~nm} \\
=-106 d B m, \mu=0.1, f=2 M H z, \lambda=1550 \mathrm{~nm} \\
=-109 d B m, \mu=0.2, f=500 \mathrm{kHz}, \lambda=1550 \mathrm{~nm} \\
=-106 \mathrm{dBm}, \mu=0.2, f=1 M H z, \lambda=1550 \mathrm{~nm}
\end{gathered}
$$

Eq. 8.2 The frequency $f$ here is the frequency of the pulsed laser and not to any photon energy. The most commonly used settings are listed above. One can see that when $\mu$ or $f$ is doubled or halved means that the attenuation has changed by +3 and $-3 \mathrm{dBm}$, respectively. See also eq 8.4

Of course, our power meter is not capable to read of values as low as these (above). It reads down to approximately $-70 \mathrm{dBm}$. Instead we read of the attenuation value $A_{\text {read }}$ on the power meter when we are not on single photon level, then we know how much to attenuate, $A_{a t t}$, the signal in order to reach the single photon level energy simply by calculating: $A_{\text {read }}-A_{\text {photon }}=A_{\text {att }}$.

If one calculates a value for $A_{\text {att }}$, after having determined $A_{\text {read }}$ and $A_{\text {photon }}$ at frequency $f_{0}$. Then, if one changes the frequency to some $f \neq f_{0}$, one does not have to recalculate $A_{a t t}$, since $A_{a t t}$ itself is frequency independent. See eq. 8.3.

$$
\begin{aligned}
& A_{\text {att }}=10 \log _{10} \frac{P_{\text {read }}}{1 \mathrm{~mW}}-10 \log _{10} \frac{P_{\text {photon }}}{1 \mathrm{~mW}}= \\
& =10 \log _{10} \frac{E_{\text {read }} f_{0}}{\mu E_{\text {photon }} f_{0}}=10 \log \frac{E_{\text {read }}}{\mu E_{\text {photon }}}
\end{aligned}
$$

Also, when one needs to increase $\mu$ from 0.1 to 0.2 or 0.3 , one just needs to decrease the signal attenuation by,

$$
\begin{aligned}
& \log _{10} \frac{E_{\text {read }}}{\frac{\mu}{2} E_{\text {photon }}}-10 \log _{10} \frac{E_{\text {read }}}{\mu E_{\text {photon }}}=10 \log _{10} 2 \approx 3 d B \\
& 10 \log _{10} \frac{E_{\text {read }}}{\frac{\mu}{3} E_{\text {photon }}}-10 \log _{10} \frac{E_{\text {read }}}{\mu E_{\text {photon }}}=10 \log _{10} 3 \approx 4.77 d B
\end{aligned}
$$




\section{List of Figures}

2.1 BB84 polarization encoding implementation, using four nonorthogonal one-photon states. . . . . . . . . . . . . . . . . . . 6

2.2 A setup of a free space Michelson interferometer. The sub-figure is showing the intensity as a function of the distance from the beam splitter to the mirror $M_{2}$, i.e $L_{1}$, (when $L_{2}$ is kept constant). Of course, for continuous light, there are infinite number of peaks as $L_{1}$ increases. . . . . . 8

2.3 A freespace Mach-Zehnder interferometer. BS, beam splitter; I, intensity detector. . . . . . . . . . . . . . . . . . . 8

2.4 QKD setup for the B92 4-state phase encoding protocol . . . . . . . . 9

3.1 Tree configuration ................... 14

3.2 Star configuration . . . . . . . . . . . . . . . . 14

4.1 Single qubit secret sharing using phase encoding. . . . . . . . . . . . 17

5.1 Error rate as a function of the distance between users. . . . . . . . . . 26

6.1 Scheme for Faraday rotation . . . . . . . . . . . . . . . . 30

6.2 One way optical fiber Mach-Zehnder interferometer. . . . . . . . . . . 32

6.3 Polarization beam splitter. . . . . . . . . . . . . . . 32

6.4 Polarization Beam Splitter attached to a Faraday Mirror . . . . . . . . 33

6.5 Alice - the interferometer. All components and interconnecting fiber inside the interferometer are polarization maintaining fiber. DVA, digital variable attenuator. We should also mention that the transmission scheme for circulator $\mathrm{C}$ is, $1 \rightarrow \mathrm{T}$ and $\mathrm{T} \rightarrow \mathrm{R}$. . . . . . . . . . . 34

6.6 Plot of expressions in eq. 6.14 . . . . . . . . . . . . . . . 37

6.7 The ideal setup for Bob, in the case with a polarization insensitive phase modulator. . . . . . . . . . . . . . . . . . . . . . 38

6.8 Bob's setup, using two polarization sensitive phase modulators. . . . . 38

6.9 Setup of Plug and Play QKD. . . . . . . . . . . . . . . . . 41

6.10 A detailed figure of the tree configuration. Notice that all users have a storage line as in Elisabeth's station. . . . . . . . . . . . . . . 43

6.11 Scheme of the Quantum Secret Sharing Plug and Play setup. . . . . . . 44 
7.1 The rise time of the TTL signal is much shorter than the rise time for the variable output. . . . . . . . . . . . . . . . . 46

7.2 An illustration of the pulse generator's different outputs. Timings and voltages should not be regarded as literary. . . . . . . . . . . . . 46

7.3 Quantum Composer Pulse Generator. . . . . . . . . . . . . . . . . . 47

$7.41550 \mathrm{~nm}$ pulsed laser with isolator and polarizer. . . . . . . . . . . 47

7.5 Optical attenuator. . . . . . . . . . . . . . . . . 48

7.6 All 5 parties, with Alice (the interferometer) in the bottom. . . . . . . 48

7.7 Fiber spools between parties. . . . . . . . . . . . . . . 49

7.8 Optical switch. . . . . . . . . . . . . . . . . . . . 49

7.9 Low-noise photo-diode. . . . . . . . . . . . . . . . . . 50

7.10 Plots over detection probability of photon and dark count as function of bias voltage of the detector. The large fluctuations at lower bias voltages in the ratio plot are simply due to low time-frame measurement relative probabilities. ................... 51

7.11 Single photon detectors. . . . . . . . . . . . . . . 51

8.1 Measurement results for the tree configuration . . . . . . . . . . . 53

8.2 Measurement results for the star configuration . . . . . . . . . . . 55

8.3 Plot over experimental data of $Q B E R_{\text {det }}$ as a function of the total distance L, from the 3 party secret sharing experiment. The solid lines correspond to theoretical values while the dots (square, triangle and circle) are the experimental values. . . . . . . . . . . . . 57

8.4 Plot over experimental data of $R_{\text {raw }}$ as a function of the total distance L, from the 3 party secret sharing experiment. The solid lines correspond to theoretical values while the dots (square, triangle and circle) are the experimental values. . . . . . . . . . . . . . . . . . 58

8.5 Plot over experimental data of $Q B E R_{\text {det }}$ as a function of the total distance $\mathrm{L}$, from the 4 party secret sharing experiment. The solid lines correspond to theoretical values while the dots (square, triangle and circle) are the experimental values. . . . . . . . . . . . . . . . 59

8.6 Plot over experimental data of $R_{\text {raw }}$ as a function of the total distance L, from the 4 party secret sharing experiment. The solid lines correspond to theoretical values while the dots (square, triangle and circle) are the experimental values. . . . . . . . . . . . . . . . 


\section{List of Tables}

1.1 Sum modulo 2 XOR operation of the binary numbers 0 and 1. . . . . 2

2.1 BB84 polarization encoding protocol using four nonorthogonal one-photon states. Announcement is made through a public channel. . . . . . . . 7

2.2 The B92 4-states phase encoding protocol . . . . . . . . . . . . 10

2.3 Sum modulo 2 operation . . . . . . . . . . . . . . . . . 11

4.1 A three party (1,2 and 3) table over some examples of qubit encoding and measurement results. . . . . . . . . . . . . . . . . . . . 17

4.2 Table over an example of the Multi-party Secret Sharing One-Time Pad 19

6.1 Scheme for a polarization beam splitter. . . . . . . . . . . . . 33

6.2 Scheme for a polarization beam splitter attached to a Faraday Mirror. . 33

6.3 Reference table for derivation below . . . . . . . . . . . . . 35

6.4 Reference table for derivation below . . . . . . . . . . . . 39

6.5 Reference table for derivation below. Regarding Elisabeth's station . . . 42

7.1 Most common settings in experiment. . . . . . . . . . . . . 45

8.1 Table of measurement results for the tree configuration with mean photon number $\mu=0.1$. Distances denote the total fiber length between Alice and user. Also, the total error rate is not being shown, instead the two main contributing factors are displayed (Visibility and $Q B E R_{\text {det }}$ ). . . . 53

8.2 Table of measurement results for the star configuration with mean photon number $\mu=0.1$. Distances denote the total fiber length between Alice and user. Also, the total error rate is not being shown, instead the two main contributing factors are displayed (Visibility and $Q B E R_{\text {det }}$ ). . . . 54

8.3 Table over distances between parties from the 3 party secret sharing experiment. L denotes the total fiber length in setup. . . . . . . . . 56

8.4 Measurement data of $Q B E R_{\text {det }}$ and visibility from the 3 party secret sharing experiment. . . . . . . . . . . . . . . 56

8.5 Measurement data of raw rates $R_{\text {raw }}$ from the 3 party secret sharing experiment. . . . . . . . . . . . . . . . . . 57

8.6 Table over distances between parties from the 4 party secret sharing experiment. L denotes the total fiber length in setup. . . . . . . . . . 58 
8.7 Measurement data of $Q B E R_{\text {det }}$ and visibility from the 4 party secret sharing experiment. . . . . . . . . . . . . . . 59

8.8 Measurement data of raw rates $R_{\text {raw }}$ from the 4 party secret sharing experiment. . . . . . . . . . . . . . . . . . . 59

8.9 Measurement data from the 5 party secret sharing experiment. . . . . . 61 


\section{Bibliography}

[1] Charles H. Bennett, Gilles Brassard: Quantum Cryptography: Public Key Distribution and Coin Tossing, International Conference on Computers, Systems \& Signal Processing. Bangalore, India (1984)

[2] A. Muller, T. Herzog, B. Huttner, W. Tittel, H. Zbinden and N. Gisin: "Plug and play" systems for quantum cryptography, Appl. Phys. Lett. Vol. 70 (1997)

[3] A. Yariv: Operator algebra for propagation problems involving phase conjugation and nonreciprocal elements, Applied Optics Vol. 26, No. 21 (1987) 4538

[4] M. Martinelli: A universal compensator for polarization changes induced by birefringence on a retracing beam, Optics Communication Vol. 72 No. 6 (1989)

[5] N. Gisin, G. Ribordy, W. Tittel, and H. Zbinden: Quantum cryptography, Reviews of Modern Physics, 74, 145-195, 2002

[6] Mark Fox: Quantum Optics, An Introduction,Oxford master series in atomic, optical and laser physics. (2006)

[7] Charles H. Bennett Quantum Cryptography Using Any Two Nonorthogonal States, Phys. Rev. Lett. Vol 68, No. 21, 3121-3124 (1992)

[8] H. Zbinden, H. Bechmann-Pasquinucii, N. Gisin, G. Ribordy: Quantum cryptography, Appl. Phys. B. Vol. 67, 743-748 (1998)

[9] M. Bourennane, F. Gibson, A. Karlsson, A. Hening, P. Johnson, T. Tsegaye, D. Ljunggren and E. Sundberg: Experiments on long wavelength (1550nm) 'plug and play' quantum cryptography systems, OPTICS EXPRESS Vol. 4, No. 10, 383-387 (1999)

[10] Donald S Bethune and William P Risk: Autocompensating quantum cryptography, New Journal of Physics 4 42.1-42.15 (2002) 
[11] G. Ribordy, J. D. Gautier, N. Gisin, O. Guinnard and H. Zbinden: Fast and user-friendly quantum key distribution Journal of Modern Optics Vol. 47, No. 2/3, 517-531 (2000)

[12] D. Stucki, O. Guinnard, G. Ribordy and H. Zbinden: Quantum key distribution over $67 \mathrm{~km}$ with a plug \& play system, New Journal of Physics 4 41.1-41.8 (2002)

[13] H. Takesue, S. W. Nam, Q. Zhang, R. H. Hadfield, T. Honjo, K. Tamaki and Y. Yamamoto: Quantum key distribution over a 40-dB channel loss using superconducting single-photon detectors, Nature Photonics Vol. 1 (2007)

[14] D. Subacius, A. Zavriev and A. Trifonov: Backscattering limitation for fiber-optic quantum key distribution systems, Appl. Phys. Lett. Vol. 86 (2005)

[15] Peter W. Shor and John Preskill: Simple Proof of Security of the BB84 Quantum Key Distribution Protocol, Phys. Rev. Lett. Vol. 85, No. 2 (2000)

[16] Mohamed Bourennane, Anders Karlsson and Gunnar Björk: Quantum Key Distribution using Multilevel Encoding: Security analysis, arxive:quant-ph/0106049 v2 12 June (2001)

[17] C. Schmidt, P. Trojek, M. Bourennane, C. Kurtsiefer, M. Zukowski and H. Weinfurter: Experimental Single Qubit Quantum Secret Sharing, Phys. Rev. Lett. Vol. 95 (2005)

[18] Mark Hillery, Vladimir Buzek and André Berthiaume: Quantum secret sharing, Phys. Rev. A. Vol. 59, No. 3 (1999)

[19] Anders Karlsson, Masato Koashi and Nobuyuki Imoto: Quantum entanglement for secret sharing and secret splitting, Phys. Rev. A, Vol. 59, No. 1 (1999)

[20] S. Gaertner, C. Kurtsiefer, M. Bourennane and H. Weinfurter: Experimental Demonstration of Four-Party Quantum Secret Sharing, Phys. Rev. Lett. 98 (2007)

[21] T. Honjo, K. Inoue, A. Sahara, E. Yamazaki, H. Takahashi: Quantum key distribution experiment through a PLC matrix switch, Optics Communications 263, 120-123 (2006)

[22] Paul Toliver et. al: Experimental Investigation of Quantum Key Distribution Through Transparent Optical Switch Elements, IEEE Photonics Technology Letters, Vol. 15, No. 11 (2003) 